\title{
DYT-TOR1A Subcellular Proteomics Reveals Selective Vulnerability of the Nuclear Proteome to Cell Stress
}

7 Departments of Neurology ${ }^{1}$, Neurobiology $^{2}$, and Cell Biology ${ }^{3}$, Duke University Medical Center 


\section{Highlights}

13 - The DYT-TOR1A nuclear proteome under cell stress showed 3-fold greater protein

14 disruptions.

15 - DYT-TOR1A MEFs show basal proteome alterations consistent with cell stress.

16 - Thapsigargin modulation of WT stress-responsive proteins is blunted in DYT-TOR1A

$17 \quad$ MEFs.

18 - TorsinB was identified as part of the cell-stress responsive proteome in WT MEFs. 


\section{Abstract}

Torsin A is a $\mathrm{AAA}^{+}$ATPase that shuttles between the ER lumen and outer nuclear

23 envelope in an ATP-dependent manner and is functionally implicated in nucleocytoplasmic

24 transport. We hypothesized that the DYT-TOR1A dystonia disease-causing variant, $\Delta \mathrm{E}$ TorsinA,

25 may therefore disrupt the normal subcellular distribution of proteins between the nuclear and

26 cytosolic compartments. To test this hypothesis, we performed proteomic analysis on nuclear and

27 cytosolic subcellular fractions from DYT-TOR1A and wildtype mouse embryonic fibroblasts

28 (MEFs). We further examined the compartmental proteomes following exposure to thapsigargin

29 (Tg), an endoplasmic reticulum (ER) stressor, because DYT-TOR1A dystonia models have

30 previously shown abnormalities in cellular stress responses. Across both subcellular

31 compartments, proteomes of DYT-TOR1A cells showed basal state disruptions consistent with

32 an activated stress response, and in response to thapsigargin, a blunted stress response. However,

33 the DYT-TOR1A nuclear proteome under Tg cell stress showed the most pronounced and

34 disproportionate degree of protein disruptions - 3-fold greater than all other conditions. The

35 affected proteins extended beyond those typically associated with stress responses, including

36 enrichments for processes critical for neuronal synaptic function. These findings highlight the

37 advantage of subcellular proteomics to reveal events that localize to discrete subcellular

38 compartments and refine thinking about the mechanisms and significance of cell stress in DYT-

39 TOR1A pathogenesis. 


\section{Cellular Stress State}

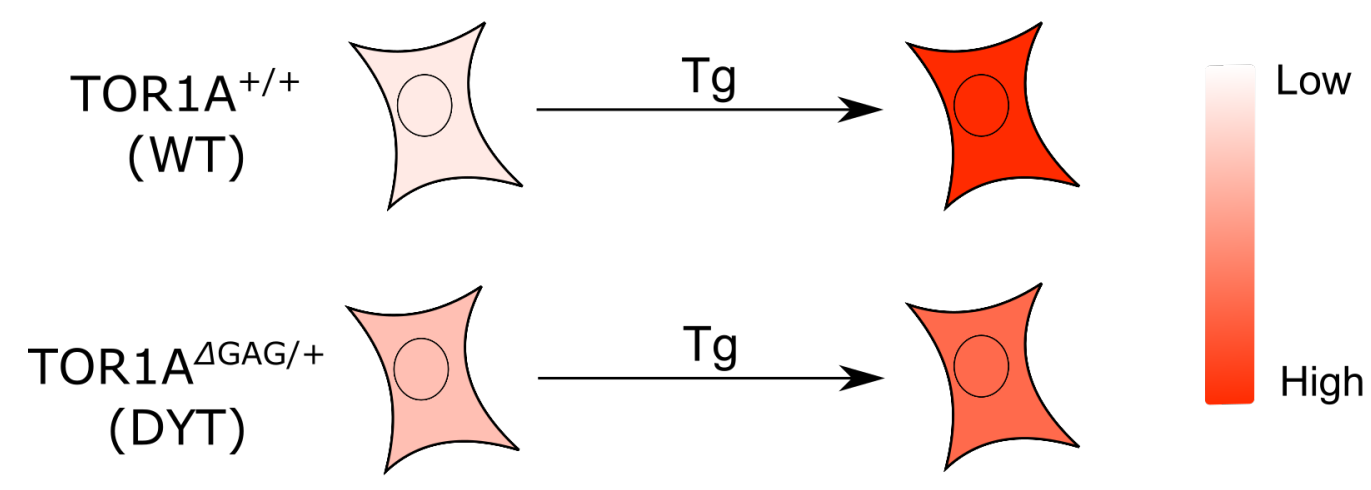

\section{Proteome Disruption Level (relative to $\mathrm{WT}$ )}

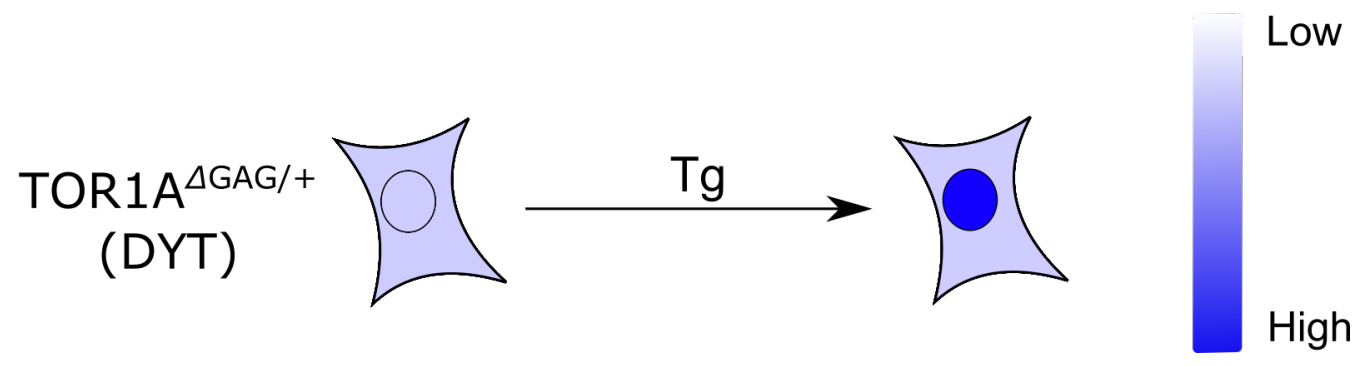




\section{$46 \quad$ Keywords}

47 Dystonia; Subcellular fractionation; TorsinA; Compartment-specific proteome; Stress Response;

48 Movement disorder

49

\section{Abbreviations}

51 Cyto, cytosolic fraction; DYT, DYT-TOR1A genotype; FC, fold change; GO, Gene Ontology;

52 LC, liquid chromatography; MEF, mouse embryonic fibroblast; MS, mass spectroscopy; Nuc,

53 nuclear fraction; RNC, relative nuclear concentration; RNP, ribonucleoprotein; Tg, thapsigargin;

54 Veh, vehicle control; WT, wildtype 


\section{Introduction}

Dystonia is a neurological movement disorder characterized by involuntary twisting and

57 abnormal postures of the limbs, trunk, and/or face (Tarsy \& Simon, 2006). Causes for dystonia are diverse, ranging from exposure to anti-psychotic medications to neurodegenerative diseases

59 (Balint et al., 2018; Bressman, 2004; Jankovic \& Tintner, 2001; van Harten et al., 1999). DYT-

60 TOR1A dystonia is a rare inherited autosomal dominant form of the disorder that is caused by an

61 in-frame trinucleotide deletion in the Torla coding sequence (n. $\Delta \mathrm{GAG}, \mathrm{p} . \Delta \mathrm{E}$ ) and leads to an early-onset, generalized dystonia (Ozelius et al., 1997).

Since the discovery of Tor la as the causal gene for DYT-TOR1A dystonia, a number of groups have characterized the function of the encoded protein, TorsinA, in its normal and mutant

66 forms. Torsin A is a member of the AAA + ATPase family of proteins. Although typically

67 Torsin A is predominantly in the lumen of the endoplasmic reticulum, perturbations preventing

68 ATP hydrolysis result in a prominent outer nuclear envelope distribution (Naismith et al., 2004).

69 These observations led to a model in which the protein shuttles between these two compartments

70 in an ATP hydrolysis-dependent manner (Naismith et al., 2004). Later studies identified two

71 TorsinA binding partners, LAP1 and LULL1, which localize to the nuclear envelope and ER

72 membrane, respectively (Chalfant et al., 2019; Chase et al., 2017; Esra Demircioglu et al., 2016;

73 Goodchild \& Dauer, 2005; M. T. Jungwirth et al., 2011; Laudermilch et al., 2016; Nery et al.,

74 2008; Saunders et al., 2017; Vander Heyden et al., 2009). Thus, it was hypothesized that TorsinA

75 likely played a role in each of these compartments. More recent studies implicate TorsinA in

76 regulation of nucleocytoplasmic transport (Chalfant et al., 2019; Ding et al., 2021; György et al.,

77 2018; Jokhi et al., 2013; Laudermilch et al., 2016; Rampello et al., 2019; VanGompel et al., 
2015). Defects in nucleoporin localization and nuclear import kinetics have been described in association with mutations in OOC-5, a TorsinA homolog in C. elegans, and in Torla in mammalian neuronal cultures and knockout mouse models (Chalfant et al., 2019; Pappas et al.,

81 2018; VanGompel et al., 2015). In addition, impaired nuclear egress functions were found in

82 both DYT-TOR1A patient fibroblasts and cultured DYT-TOR1A mouse neurons (György et al.,

83 2018). These defects may be related to TorsinA's role in regulating nuclear budding, a process

84 essential for nuclear egress of mega-ribonucleoproteins (megaRNPs) (Jokhi et al., 2013; Speese

85 et al., 2012). Collectively, these findings suggest that TorsinA plays a role in regulating nucleocytoplasmic transport, and that the DYT-TOR1A-associated TorsinA mutation, $\Delta \mathrm{E}$,

87 impairs these functions.

In addition to nucleocytoplasmic transport defects, our lab and several others have found

90 that multiple DYT-TOR1A dystonia model systems exhibit altered cellular stress response

91 pathways (Beauvais et al., 2016, 2018; Chen et al., 2010; Cho et al., 2014; Nery et al., 2011;

92 Rittiner et al., 2016; Zacchi et al., 2014; Zhao et al., 2016). It is currently unknown how the

93 DYT-TOR1A mutation specifically causes these cellular stress response defects. However,

94 alleviation of these defects by pharmacological and genetic approaches has been shown to

95 improve DYT-TOR1A associated cellular phenotypes (Rittiner et al., 2016), suggesting that

96 cellular stress responses may play an important role in DYT-TOR1A disease pathophysiology.

99 TOR1A causative mutation in whole cell and tissue lysate preparations (Beauvais et al., 2016,

100 2018; Martin et al., 2009). These approaches have successfully identified disease model- 
101 associated protein defects. However, because many subcellular compartments such as the

102 nucleus comprise only a proportionally small part of the total proteome, compartment-specific

103 defects are likely to be overlooked by such approaches. Recent studies of amyotrophic lateral

104 sclerosis demonstrate the potential for nucleocytoplasmic proteomic analyses by identifying

105 nucleus-specific defects in RNA transport and cytosol-specific defects in protein translation and

106 folding (J. E. Kim et al., 2017; Ortega et al., 2020). In the present study, we adopt a similar

107 nucleocytoplasmic fractionation technique alongside quantitative proteomics to address whether

108 the DYT-TOR1A mutation causes subcellular compartment-specific proteomic disruptions.

109

Our results identify compartment- and stress-specific disruptions associated with the

111 DYT-TOR1A genotype that include disruptions of proteins that are normally cell stress

112 modulated and of TorsinA and TorsinB levels and localization. The most striking result however

113 was that, despite our leading hypothesis that defects in nucleocytoplasmic transport might affect

114 both compartments, the DYT-TOR1A mutation was found to cause the most pronounced and

115 disproportionate insult to the nuclear proteome and selectively under cell stress. This result

116 indicates that $\Delta \mathrm{E}$ Torsin $\mathrm{A}$ causes a particular vulnerability to the integrity of the nuclear

117 proteome in the face of cellular stressors.

119 Materials and Methods

120 Animals

DYT-TOR1A knock-in mice (Tor $\left.1 a^{\Delta \mathrm{GAG} /+}\right)$ (courtesy of Dr. W. Dauer, University of

122 Michigan) (Goodchild et al., 2005) on C57BL/6 background were bred in standard housing 
123 conditions with food and water provided ad libitum. All procedures were approved by the Duke

124 University Institutional Animal Care and Use Committee (IACUC).

125

Mouse Embryonic Fibroblast (MEF) Extraction, Isolation, and Immortalization

on a $\mathrm{C} 57 \mathrm{BL} / 6$ background. MEF extraction occurred with minor modifications from the protocol as described in (Jozefczuk et al., 2012). Three DYT-TOR1A MEF lines and three WT MEF lines

131 were produced from littermates of a single litter. The pregnant dam was euthanized at

132 approximately 14 days post-coitum using isoflurane followed by decapitation. The uterine horns

133 were dissected out and rinsed in 70\% (v/v) ethanol and PBS (Gibco, Invitrogen) before placing

134 into a Petri dish containing PBS (Gibco, Invitrogen). Each individual embryonic sac was

135 separated from the uterine horns and placenta, and then placed into a separate dish containing

136 PBS. Each embryo was dissected out of the embryonic sac and its head and red organs were

137 removed. The remaining embryonic tissue was placed into a clean Petri dish where it was minced

138 with a sterile razor blade. $1 \mathrm{~mL}$ of $0.05 \%$ trypsin/EDTA (Gibco, Invitrogen) was added to each

139 dish. The mixture was transferred into a $15 \mathrm{~mL}$ Falcon tube and incubated at $37^{\circ} \mathrm{C}$ for 30

140 minutes. After each 10 minutes of incubation, MEFs were dissociated via pipetting. Trypsin was

141 inactivated by adding $2 \mathrm{~mL}$ of fetal bovine serum-containing media ("MEF media" described in

142 Cell Culture section below) to each tube. The MEFs were then centrifuged at $500 \mathrm{x} \mathrm{g}$ for 5

143 minutes. The supernatant was removed, and the cell pellet was resuspended in $10 \mathrm{~mL}$ of warm

144 MEF media. This solution was then plated on TC dishes coated in 1\% Matrigel (Corning). After 
1452 passages, the MEFs were genotyped and subsequently immortalized via the SV40 T antigen as 146 described in (H. Harding, 2003). Cell lines were used within 5 passages.

148 Genotyping

All genotyping was conducted as previously described in (Goodchild et al., 2005).

\section{Cell Culture}

MEFs were grown in MEF media which consisted of $500 \mathrm{~mL}$ of DMEM, high glucose, 153 pyruvate (Thermo Fisher, \#11995), $50 \mathrm{~mL}$ of Fetal Bovine Serum, $5 \mathrm{~mL}$ of Antibiotic-

154 Antimycotic (Gibco, Invitrogen), $5 \mathrm{~mL}$ of $200 \mathrm{mM} \mathrm{L-Glutamine} \mathrm{(Gibco,} \mathrm{Invitrogen),} 5 \mathrm{~mL}$ of

155 MEM Non-Essential Amino Acids Solution (Gibco, Invitrogen), and $500 \mu \mathrm{L}$ of 2-

156 Mercaptoethanol (Sigma-Aldrich). MEFs were grown in incubators at $37{ }^{\circ} \mathrm{C} / 5 \% \mathrm{CO}_{2}$.

\section{Thapsigargin Treatment and Subcellular Fractionation}

Three separate experiments were performed exposing MEFs to the cell stressor,

160 thapsigargin (Tg). During each experiment, a pair of MEF lines (1 WT and 1 DYT-TOR1A) was

161 treated with either $1 \mu \mathrm{M}$ Tg dissolved in DMSO or an equivalent volume of DMSO (Vehicle

162 control, Veh). After six hours of treatment at $37^{\circ} \mathrm{C}$, the MEFs were subcellularly fractionated

163 into nuclear and cytosolic fractions. MEFs for subcellular fractionation were acquired through

164 trypsinization from $90 \%$ confluent TC plates. Subcellular fractionation of MEFs was then carried

165 out as described in (Suzuki et al., 2010). Briefly, the procedure involves a weak and brief

166 detergent extraction ( $0.01 \% \mathrm{NP} 40,3 \mathrm{~min}$, , room temperature), centrifugation to collect the

167 supernatant (cytosolic fraction) and then further solubilization in the same buffer alongside 
168 sonication to penetrate the double bilayer membranous nuclear compartment, with a second 169 centrifugation and supernatant collection for the nuclear fraction.

171 Western Blotting

172 Lysates for Western analysis were produced either through the subcellular fractionation

173 protocol described earlier or via a whole-cell lysate produced with RIPA buffer-induced cell

174 lysis. Protein content from each lysate was determined via Bicinchoninic Acid (BCA) assay.

175 Samples were prepared such that each sample contained an equal mass of protein and $1 \mathrm{x}$

176 Laemmli buffer. Samples were reduced and denatured with 2-mercaptoethanol and incubated at

$17797^{\circ} \mathrm{C}$ for 5 minutes. Equal volumes of sample were loaded into the wells of an SDS-PAGE gel

178 along with a protein ladder. After 45 minutes electrophoresis at $175 \mathrm{~V}$, the protein within the gel

179 was transferred to a nitrocellulose membrane.

180

181 Following transfer, the membrane was blocked in 5\% BSA solution prepared in TBST for

1821 hour at room temperature. The membrane was then incubated overnight on a shaker at $4{ }^{\circ} \mathrm{C}$ in

183 blocking solution amended with the primary antibody [anti-Lamin B1 (Abcam; ab16048;

184 1:1000), anti-GAPDH (Abcam; ab9485; 1:1000), anti-Na+/K+ - ATPase (Santa Cruz

185 Biotechnology; sc-21712; 1:500), anti-BiP (Santa Cruz Biotechnology; sc-13968; 1:500)].

186 Following primary incubation, the membrane was washed three times with TBST for 5-10

187 minutes each time. The membrane was then re-blocked in blocking solution for 1 hour.

188 Membranes were then placed in blocking solution with secondary antibody [Alexa Fluor 790

189 Goat anti-Rabbit and/or Alexa Fluor 680 Goat anti-Mouse (Thermo Fisher)] at a dilution of

190 1:1000. The membrane was incubated in the secondary solution for 1 hour before being washed 
191 three times in TBST for 5-10 minutes per wash. The membrane was imaged on a LI-COR

192 Odyssey Imaging System. The visualized bands were quantified using ImageJ.

193

194 Immunofluorescent Staining

MEF lines (three WT and three DYT-TOR1A) were plated into individual wells on a 96well plate, such that each line was plated into eight wells. Four of the wells for every line were treated with Veh and the other four wells for each line were treated with $1 \mu \mathrm{M}$ Tg for six hours.

198 Following treatment, the wells were fixed with 4\% paraformaldehyde, permeabilized and

199 blocked with blocking solution (0.1\% Triton-X 100 in PBS, 1\% bovine serum albumin, 10\%

200 normal donkey serum) for 1 hour. The wells were then stained with a primary antibody [anti-

201 Tbce (Thermo-Fisher, PA5-100346, 1:200), anti-Pds5B (Thermo-fisher; PA5-59029; 1:500)]

202 overnight at $4^{\circ} \mathrm{C}$. Following three washes with wash buffer solution $(0.1 \% \mathrm{BSA}$ in PBS),

203 secondary staining was conducted using Hoechst 33342 (MilliporeSigma; 1:1000) to stain the

204 nucleus and donkey Anti-rabbit Alexa Fluor 488 (Life Technologies; 1:1000) for 1 hour at room

205 temperature. Following three additional washes with wash buffer solution, the wells were filled

206 with dilution buffer (1\% BSA, 1\% normal donkey serum, 0.3\% Triton X-100, and 0.01\% sodium

207 azide in PBS). Sixteen imaging fields from each well were acquired at a magnification of 20x

208 from a Thermofisher CX5 HC imager. Images were acquired in both the blue and green channel

209 to identify the cell nuclei and quantify the protein of interest. Following image acquisition,

210 images were analyzed by CellProfiler 3.1.9 (McQuin et al., 2018). 


\section{Immunofluorescence Quantification and Data Analysis}

215 Nuclei were identified via the blue channel Hoechst stain. The cytosol was identified via

216 the green channel protein immunofluorescence stain and nuclear position information as

217 determined from the blue channel Hoechst stain. Nuclear fluorescence was quantified by

218 integrating the intensity of the green channel protein immunofluorescence across pixels

219 identified as being part of the nucleus. Cytosolic fluorescence was quantified by integrating the

220 intensity of the green channel protein immunofluorescence across each pixel within the region

221 identified as being part of the cytosol.

222

223 Puncta were identified via a modified speckle counting pipeline developed by

224 CellProfiler (McQuin et al., 2018). Analyses were conducted on image masks containing only

225 the nuclei, as well as image masks containing only the cytosol to quantify puncta in each of the

226 subcellular compartments. Puncta frequency was determined by taking the number of speckles

227 identified per image and then dividing by the number of nuclei or cytosolic areas within that

228 image. Puncta intensity was determined by integrating the intensity of all the puncta within the

229 field and dividing by the number of nuclei or cytosolic areas within that image.

Each quantified value (nuclear fluorescence, cytosolic fluorescence, puncta frequency,

232 puncta intensity) was calculated for each of the sixteen fields imaged per well and these sixteen

233 values were averaged to produce a single mean value for each well. Unpaired t-tests were used to

234 compare mean values from the four biological replicate wells across both genotypes with and

235 without stress treatment. 


\section{Quantitative LC/MS/MS and Proteomic Analysis}

Twenty-four samples in total were submitted to the Duke Proteomics and Metabolomics

Shared Resource (two subcellular fractions from each of the six MEF lines treated with either Tg or Veh). While the fractions were collected over three separate cell culture experiments, they were all analyzed within a single liquid chromatography with tandem mass spectroscopy casein at a total of 20,30 , or $40 \mathrm{fmol} / \mu \mathrm{g}$, then reduced with $10 \mathrm{mM}$ dithiothreitol for $30 \mathrm{~min}$ at $80^{\circ} \mathrm{C}$, and alkylated with $20 \mathrm{mM}$ iodoacetamide for $30 \mathrm{~min}$ at room temperature. Next, they

245 were supplemented with a final concentration of $1.2 \%$ phosphoric acid and $741 \mu \mathrm{L}$ of S-Trap

246 (Protifi) binding buffer $(90 \% \mathrm{MeOH} / 100 \mathrm{mM}$ TEAB). Proteins were trapped on the S-Trap,

247 digested using $20 \mathrm{ng} / \mu \mathrm{L}$ sequencing grade trypsin (Promega) for 1 hour at $47^{\circ} \mathrm{C}$, and eluted using

$24850 \mathrm{mM}$ TEAB, followed by $0.2 \% \mathrm{FA}$, and lastly using $50 \% \mathrm{ACN} / 0.2 \% \mathrm{FA}$. All fractions were

249 then lyophilized to dryness and resuspended in $20 \mu \mathrm{L} \mathrm{1 \%} \mathrm{TFA/2 \%} \mathrm{acetonitrile} \mathrm{containing} 12.5$

$250 \mathrm{fmol} / \mu \mathrm{L}$ yeast alcohol dehydrogenase (ADH_YEAST). Three QC Pools were created: 1) $3 \mu \mathrm{L}$

251 from each of the nuclear fractions, 2) $3 \mathrm{uL}$ from each of the cytosolic fractions 3) $3 \mu \mathrm{L}$ from each

252 of all of the fractions, both nuclear and cytosolic. All QC Pools were run periodically randomly

253 interspersed throughout the test fractions.

257 accurate mass tandem mass spectrometer (Thermo) via a nanoelectrospray ionization source.

258 Briefly, the fraction was first trapped on a Symmetry C18 $20 \mathrm{~mm} \times 180 \mu \mathrm{m}$ trapping column $(5$

$259 \mu \mathrm{L} / \mathrm{min}$ at $99.9 / 0.1 \mathrm{v} / \mathrm{v}$ water/acetonitrile), after which the analytical separation was performed 
260 using a $1.8 \mu \mathrm{m}$ Acquity HSS T3 C18 $75 \mu \mathrm{m} \times 250 \mathrm{~mm}$ column (Waters Corp.) with a 90-min

261 linear gradient of 5 to $30 \%$ acetonitrile with $0.1 \%$ formic acid at a flow rate of 400

262 nanoliters/minute $(\mathrm{nL} / \mathrm{min})$ with a column temperature of $55^{\circ} \mathrm{C}$. Data collection on the Fusion

263 Lumos mass spectrometer was performed in a data-dependent acquisition (DDA) mode of

264 acquisition with a r=120,000 (@ m/z 200) full MS scan from m/z 375 - 1500 with a target AGC

265 value of 2e5 ions. MS/MS scans were acquired at Rapid scan rate (Ion Trap) with an AGC target

266 of $5 \mathrm{e} 3$ ions and a max injection time of 100 milliseconds. The total cycle time for MS and

267 MS/MS scans was 2 seconds. A 20s dynamic exclusion was employed to increase depth of

268 coverage. The total analysis cycle time for each fraction injection was approximately 2 hours.

Following 35 total UPLC-MS/MS analyses (excluding conditioning runs, but including 3

271 replicate QC Pool, 4 replicate nuclear and 4 replicate cytosolic Pool injections), data was

272 imported into Proteome Discoverer 2.2 (Thermo Scientific Inc.), and analyses were aligned

273 based on the accurate mass and retention time of detected ions ("features") using Minora Feature

274 Detector algorithm in Proteome Discoverer. Protein levels are reported in arbitrary units (a.u.)

275 based on the relative peptide abundance measures which were calculated by area-under-the-

276 curve (AUC) of the selected ion chromatograms of the aligned features across all runs. The

277 MS/MS data was searched against the SwissProt M. musculus database (downloaded in Apr

278 2017) and an equal number of reversed-sequence "decoys" for false discovery rate

279 determination. Mascot Distiller and Mascot Server (v 2.5, Matrix Sciences) were utilized to

280 produce fragment ion spectra and to perform the database searches. Database search parameters

281 included fixed modification on Cys (carbamidomethyl) and variable modifications on Meth

282 (oxidation) and Asn and Gln (deamidation). Peptide Validator and Protein FDR Validator nodes 
283 in Proteome Discoverer were used to annotate the data at a maximum 1\% protein false discovery 284 rate.

287 manner. If less than half of the values are missing across all samples, values are imputed with an

288 intensity derived from a normal distribution defined by measured values within the same

289 intensity range (20 bins). If greater than half values are missing for a peptide across all samples

290 and a peptide intensity is $>5 \mathrm{e} 6$, then it was concluded that peptide was misaligned and its

291 measured intensity is set to 0 . All remaining missing values are imputed with the lowest $5 \%$ of

292 all detected values. All analyses presented here are based on these normalized values. The

293 complete proteomic dataset has been deposited with Mendeley data and is further detailed in a

294 Data In Brief accompanying article.

\section{Data Analysis and Statistical Analysis}

298 Throughout the data collection phase of the study, cell genotype and stress-treatment

299 conditions were blinded variables. Unblinding occurred upon return of processed proteomic data.

$301 \quad$ For proteomic data analysis, proteins represented by only a single peptide were removed

302 from the data set prior to further analysis to reduce the number of Type 1 errors. Unpaired t-test

303 p-values and fold changes for each protein were calculated for each comparison. P-values and

304 fold changes were calculated using GSEA software from the Broad Institute via a Student's t-test

305 (Mootha et al., 2003; Subramanian et al., 2005). Proteins that showed an uncorrected p-value less 
than 0.05 and a DYT-TOR1A/WT or WT/DYT-TOR1A ratio greater than 1.5 (DYT-

307 TOR1A/WT fold change greater than $\left.\pm \log _{2}(0.585)\right)$ were considered as the "top hits" for further

308 analysis. Metascape was used to conduct a Gene Ontology analysis on the top hits (Zhou et al.,

309 2019). Top hits were analyzed using the entire discovered proteome as the background to

310 consider for enrichment.

All other statistical testing used unpaired t-tests calculated by GraphPad Prism version

313 8.3.1 for MacOS unless otherwise indicated.

\section{Results}

\section{Subcellular Fractionation of Mouse Embryonic Fibroblasts (MEFs)}

318 (genotype hereafter abbreviated as DYT-TOR1A or DYT) and wildtype (WT) littermate

319 embryos according to standard methodology (Methods). Cultures from 3 independent lines for

320 each genotype were grown to confluence and then treated with either $1 \mu \mathrm{M}$ thapsigargin (Tg) or

321 vehicle (Veh) for six hours prior to harvesting (Fig. 1A). Nuclear and cytosolic cellular fractions

322 were prepared according to previously described methods based on serial exposure to a mild

323 detergent extraction that does not significantly solubilize nuclear membranes, but is sufficient to

324 penetrate plasma membrane and enable extraction of cytosolic components and organelles,

325 followed by a nuclear membrane-solubilizing sonication step (Suzuki et al., 2010). Western

326 analysis was performed to confirm that markers for the nuclear (Lamin B1) and the cytosolic

327 (GAPDH, BiP, Na,K-ATPase $\alpha 1$ ) subcellular compartments were differentially distributed in the

328 fractions as predicted in both genotypes (Fig. 1B and Fig. S1). Nuclear and cytosolic fractions 
329 prepared from 3 independent cell lines for each genotype were then analyzed by quantitative

330 liquid chromatography-tandem mass spectrometry (LC/MS/MS).

A total of 4801 proteins were detected across all samples. Of those, 3921 proteins had at

333 least two distinct peptides and mapped to a unique mouse gene identifier using GSEA software;

334 these proteins were used for subsequent analyses. Over $90 \%$ of proteins were identified in both

335 subcellular fractions and treatment conditions (Fig. S2A). Recognizing that most proteins are

336 present in both subcellular compartments to varying degrees, we next calculated the relative

337 nuclear concentration $(\mathrm{RNC})$ (nuclear level/total $\mathrm{nuclear}_{+}$cytosolic) for each of the 3921 proteins. The

338 RNC has been used previously to characterize the proteome and demonstrated only a small

339 fraction of proteins being almost exclusive to nuclear or cytosolic fractions, with a majority of

340 proteins having intermediate RNC values (Wühr et al., 2015). The nucleocytoplasmic

341 distribution of proteins in our samples is consistent with those prior observations and was similar

342 across the two genotypes (Fig. 1C). In addition, we performed standard bioinformatic analysis

343 using Gene Ontology (GO) on the nuclear and cytosolic fraction proteomic datasets to determine

344 whether enrichments characteristic of nuclear and cytosolic components were detected in the

345 corresponding fractions (For ease of presentation, results of both genotypes were combined.

346 Individual genotype analyses yielded similar conclusions, data not shown). The top GO terms

347 associated with proteomics of the cytosolic fractions included "cytosolic part" (Fig. 1D). In

348 addition to cytosolic proteins, we also observed enrichment for cytosolic vesicle membrane

349 proteins as shown by the strong enrichment of the GO terms "membrane coat" and "Ficolin-1

350 rich granule lumen". Conversely, the top GO terms associated with nuclear fractions included

351 "nuclear body" (Fig. 1E). We further noted that GO analysis of the nuclear fraction also included 
352 "mitochondrial membrane part" suggesting that mitochondria may be preferentially extracted

353 with the nuclear fraction. Together, these characterizations establish that components of the

354 nucleus and cytosol are relatively enriched in the nuclear and cytosolic fractions, respectively.

A

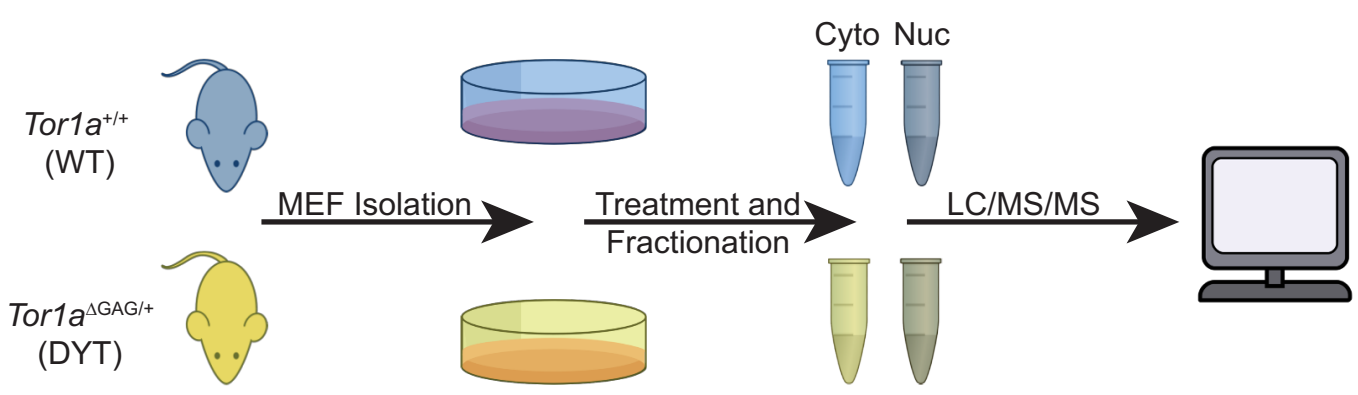

B

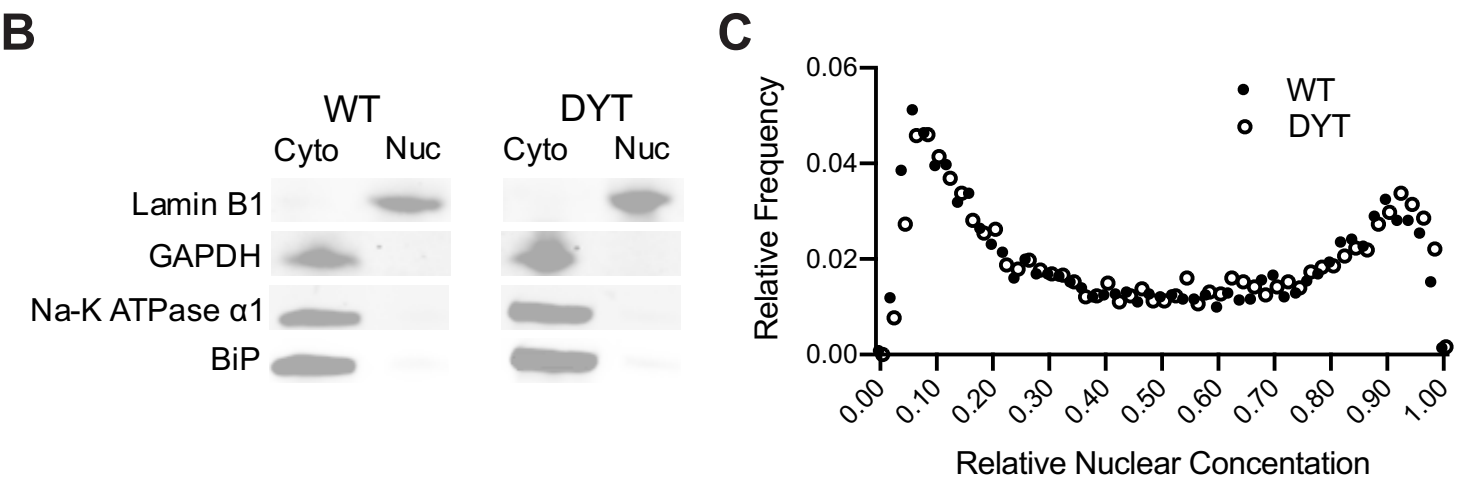

Lamin B1

GAPDH

Na-K ATPase $\alpha 1$

BiP

C

D
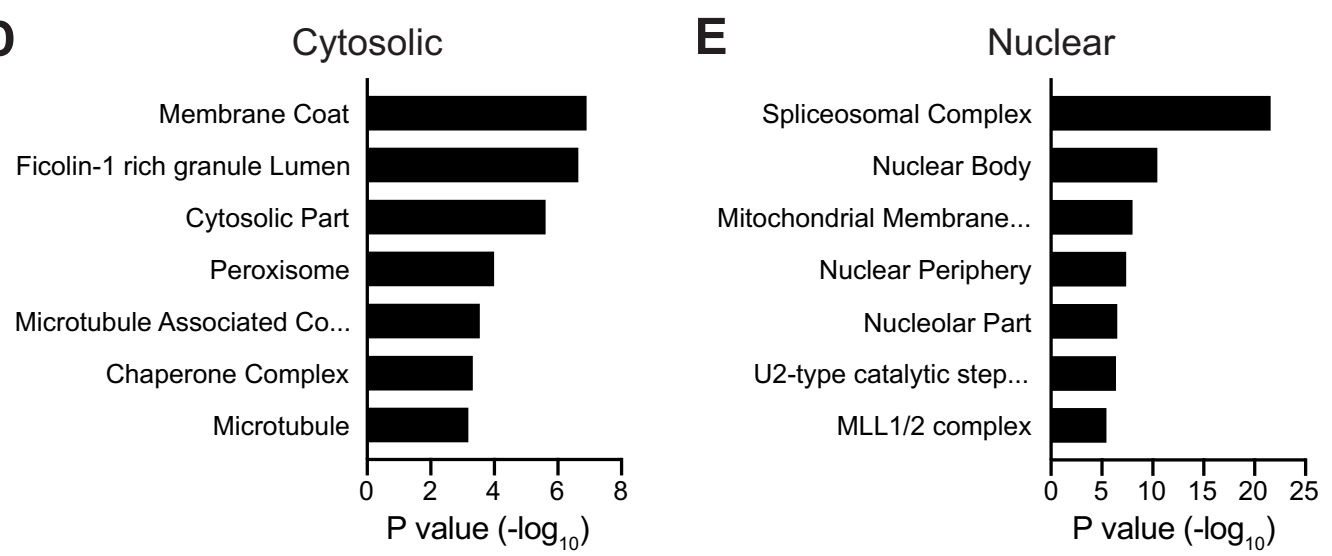

357 Fig. 1. Subcellular fractionation enriches for cytosolic and nuclear components similarly in both

358 WT and DYT-TOR1A cell lines. (A) Experimental design schematic. Mouse embryonic 
360 mice and wildtype litter mates. MEF lysates were fractionated into cytosolic (Cyto) and nuclear

361 (Nuc) fractions and then subjected to quantitative differential proteomics analysis. (B)

362 Representative Western blots of subcellular fraction markers in WT and DYT-TOR1A cell lines.

363 The nuclear membrane marker, Lamin B1, is predominately sequestered in the nuclear fraction

364 while GAPDH, Na-K ATPase $\alpha 1$, and BiP (cytosolic, plasma membrane, and endoplasmic

365 reticulum resident proteins, respectively) are enriched in the cytosolic fraction. (C) Frequency

366 distribution of calculated relative nuclear concentration (RNC) values across the entire proteome

367 from WT (solid circles) and DYT-TOR1A (open circles) samples. RNC values are calculated for

368 each individual protein by taking the protein abundance within the nuclear fraction and dividing

369 by the sum of the nuclear and cytosolic fraction protein abundances (adapted from Wühr et al.,

370 2015). (D-E) Gene Ontology analysis of proteins enriched within the nuclear (D) and cytosolic

371 (E) fraction (dataset of combined genotypes using threshold of $\mathrm{p}<0.001$ by $\mathrm{t}$-test).

\section{DYT-TOR1A Genotype-Dependent Subcellular Proteome Differences}

To identify proteome differences caused by the DYT-TOR1A genotype, an average fold

375 change (FC) and p-value were calculated for each protein by comparing levels between WT and

376 DYT-TOR1A samples under basal conditions $(\mathrm{Veh})(\mathrm{n}=3$ independent biological replicates per

377 genotype). Using thresholds of an uncorrected p-value of less than 0.05 and fold change of \pm 1.5

378 FC, which corresponds to a 50\% increase in DYT-TOR1A levels relative to WT or WT levels

379 relative to DYT-TOR1A, we identified 152 proteins with genotype-dependent differences in the

380 cytosolic fractions (Fig. 2A). A similar number of differences were identified in the nuclear

381 fractions (Fig. 2B). There was less than 3\% overlap between the differentially affected proteins

382 in the nuclear and cytosolic samples (Fig. S2B). Gene Ontology analysis of differentially 
383 affected proteins revealed enrichment of proteins associated with mitochondrion organization

384 and ATP metabolism (Fig. S3). This GO term enrichment was present in both the nuclear and

385 cytosolic DYT-TOR1A-disrupted protein datasets (Fig. S3). These results are consistent with

386 processes that have been previously implicated in DYT-TOR1A dystonia (Beauvais et al., 2016;

387 Martin et al., 2009).

Prior studies have also described numerous cellular stress response defects in DYT-

390 TOR1A dystonia models (Beauvais et al., 2016; Chen et al., 2010; C. E. Kim et al., 2010; Nery

391 et al., 2011; Rittiner et al., 2016; Zhao et al., 2016). Because many of these defects are apparent

392 only following stress treatment, we additionally performed the proteomic experiment in samples

393 after six hours of thapsigargin ( $\mathrm{Tg}$ ) exposure, a compound that causes cell stress by releasing

394 internal calcium stores. With Tg treatment, we found that cytosolic samples showed a similar

395 number of genotype-dependent differences as non-stressed (Veh) samples (115 proteins) (Fig.

396 2C). However, in striking contrast to cytosolic fractions, Tg treatment caused a greater than 3-

397 fold increase in genotype-dependent differences in the nuclear proteome (624 proteins) relative

398 to the non-stressed samples (187 proteins) (Fig. 2D). These data reveal that among cytosolic and

399 nuclear compartments in basal and stressed states, the DYT-TOR1A genotype most severely

400 disrupts the composition of the nuclear proteome and does so selectively in the presence of a 401 cellular stressor.

Given the similarity in the number of protein differences between the cell stress and basal

404 conditions in the cytosolic fractions, we next examined the extent to which genotype-dependent

405 differences were due to the same proteins being affected in multiple conditions. Surprisingly, 
406 there was little overlap - with less than $10 \%$ of proteins being shared between the stressed and

407 basal state conditions (Fig. S2B). These findings indicate that, in both the nuclear and cytosolic

408 fractions, the proteome disruptions caused by DYT-TOR1A under cell stress affects proteins that

409 are largely distinct from those in the basal state.

Next, we evaluated genotype-dependent differences in the subcellular fractionation of

412 TorsinA itself. Multiple prior studies have found that the DYT-TOR1A mutation of Torla $(\Delta \mathrm{E}$

413 TorsinA) drives TorsinA mislocalization from the ER to the nuclear envelope (Bragg et al.,

414 2004; Calakos et al., 2010; Gonzalez-Alegre \& Paulson, 2004; Goodchild \& Dauer, 2004;

415 Hewett et al., 2000; Kustedjo et al., 2000; Liang et al., 2014; Naismith et al., 2004; Torres et al.,

416 2004). The results for TorsinA were not included in our proteomic analysis because TorsinA was

417 identified by only a single unique identifying peptide, and this is associated with an increased

418 risk for misidentifying proteins (Carr et al., 2004). However, given the particular relevance of

419 Torsin A data to this study, we used the single peptide data to examine its distribution between

420 nuclear and cytosolic compartments. We found that relative to WT samples, TorsinA levels were

421 significantly lower in cytosolic fractions of DYT-TOR1A samples $(p=0.004)$ and that there was

422 also a non-significant trend toward higher levels of TorsinA in nuclear fractions of DYT-TOR1A

423 samples $(\mathrm{p}=0.232)$ significantly altering its distribution to be biased towards the nuclear

424 compartment (N/C ratio) (Fig. 2E).

425

Interpretation of data from our approach and most other subcellular proteomic

427 approaches relies upon the assumption that cell constituents fractionate normally (Ortega et al., 428 2020; Tribl et al., 2005). In Figure 1B-C, we observed that there were no obvious solubilization 
429 differences between WT and DYT-TOR1A MEFs. Nonetheless, for any specific protein of

430 interest, the use of an orthogonal methodology would be a desirable validation step. To date,

431 conventional immunofluorescence has not revealed the TorsinA redistribution in genetic

432 construct-valid DYT-TOR1A cells that we detected here using quantitative proteomics. To

433 better understand the misdistribution of $\Delta \mathrm{E}$ Torsin $\mathrm{A}$ and address the integrity of nuclear

434 envelope partitioning in DYT-TOR1A cells, we evaluated the partitioning of known nuclear

435 envelope proteins, the LINC complexes in WT and DYT-TOR1A MEFs (Fig. S4). We found

436 that LINC proteins present in our datasets enriched in the nuclear fraction as expected and

437 partitioned similarly in WT and DYT-TOR1A samples. We also present two examples of

438 conventional ICC validation. Levels of Tbce and Pds5b were significantly altered in DYT-

439 TOR1A in the nuclear compartment under Tg cell stress condition (Fig. S5-S6). Proteomic

440 analysis of Tbce showed a compartment-specific decrease in the Tg-nucleus and no difference in

441 cytosolic levels (Fig. S5B, D); findings which were replicated with conventional ICC (Fig. S5C,

442 E). Pds5b levels were significantly increased in DYT-TOR1A Tg-nuclear samples (Fig. S6B).

443 Using ICC, nuclear Pds5b immunostaining intensity was significantly different by genotype;

444 however, instead of increased, Pds5b staining was significantly decreased in DYT-TOR1A (Fig.

445 S6C). Interestingly, in WT cells, Pds5b was more commonly in strongly staining puncta, raising

446 the possibility that reduced solubility of punctate Pds $5 \mathrm{~b}$ might give rise to the proteomic result of

447 lower levels in WT cells (Fig. S6D, E). To summarize, while ICC for both proteins confirmed

448 genotype effects, these two examples highlight the range of disruptions that might underlie the

449 proteomic results. 

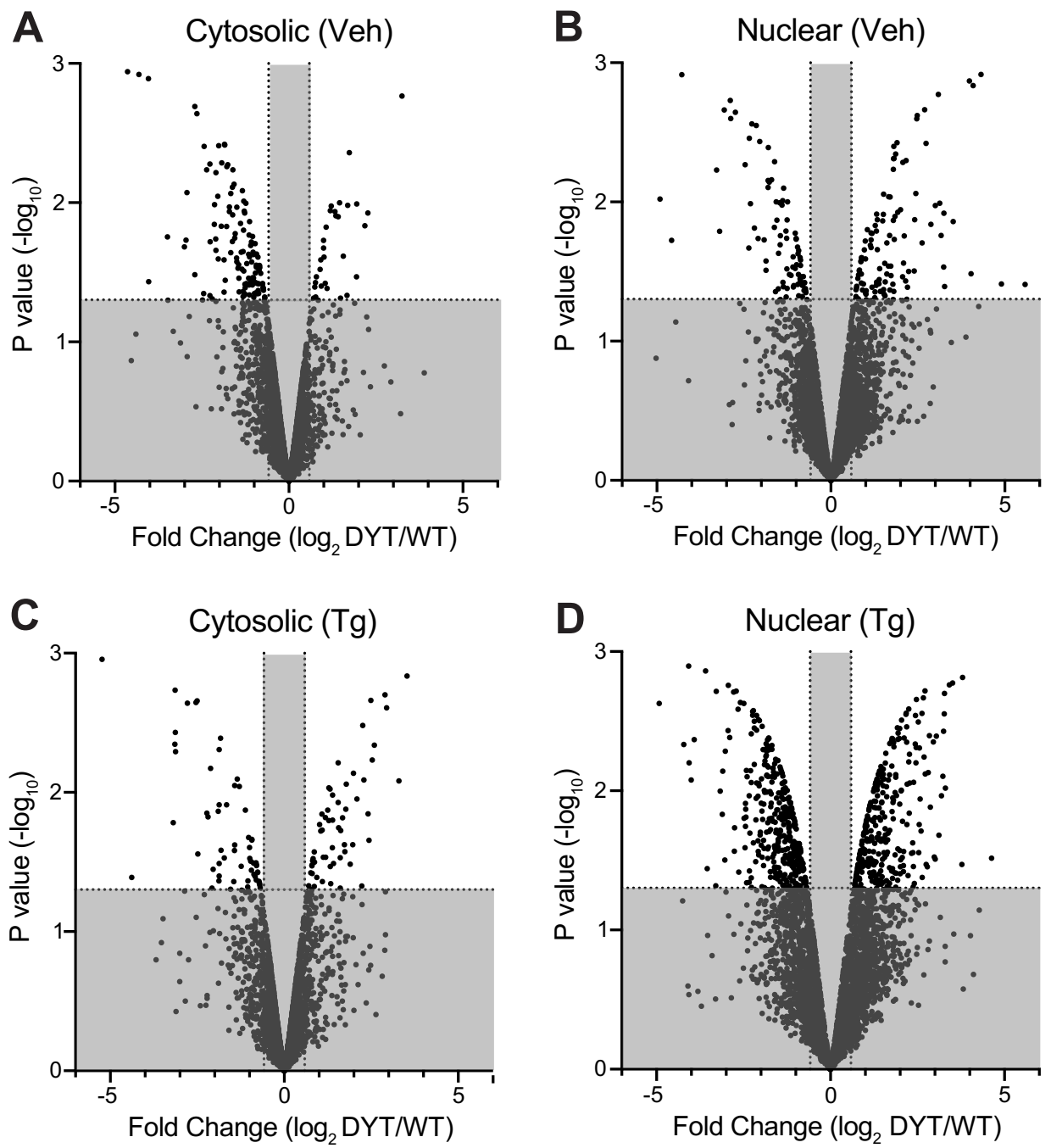

$\mathbf{E}$ Cytosolic

Nuclear

N/C Ratio
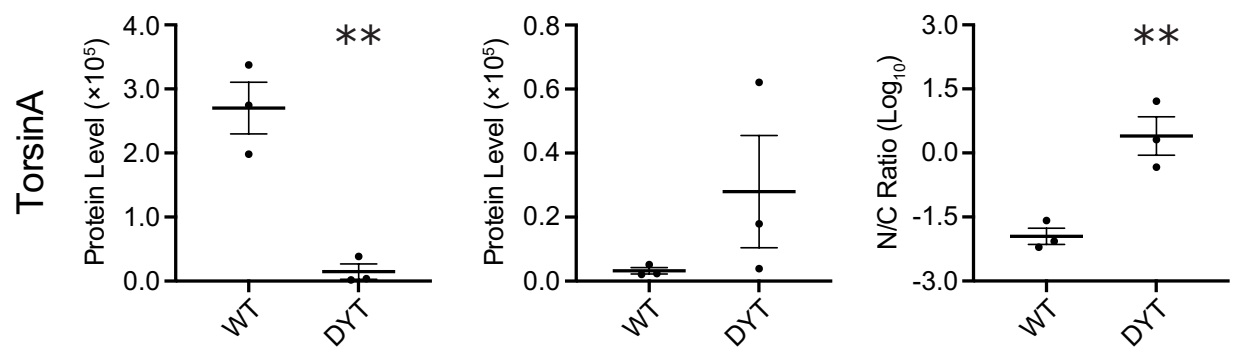

451 Fig. 2. Compartment-specific proteome disruptions associated with DYT-TOR1A MEFs. (A-B)

452 Volcano plots showing proteome-wide differences in protein abundances between DYT-TOR1A

453 and WT cytosolic (A) and nuclear (B) fractions under basal conditions (Veh). Differences in 
454 protein abundance are represented as fold change (using $\log _{2}$ transform) and p-value is calculated

455 by unpaired t-test for each protein ( $\mathrm{n}=3$ biological replicates). Horizontal dashed line indicates $\mathrm{p}$ -

456 value less than 0.05 . Vertical dashed lines indicate fold changes of \pm 1.5 . (C-D) Corresponding

457 volcano plots for fractions from thapsigargin-treated (Tg) cells. (E) Relative TorsinA peptide

458 levels (a.u.) in cytosolic (Veh) and nuclear (Veh) fractions and the nuclear:cytosolic (N/C) ratio.

459 Error bars indicate S.E.M.. For all comparisons, $\mathrm{n}=3$ biological replicates, $\mathrm{p}$-value determined

460 by unpaired t-test $(* * \mathrm{p}<0.01)$.

\section{Thapsigargin Stress-Responsive Proteins in WT MEFs}

Thus far, our proteomic analyses reveal that the largest DYT-TOR1A genotype-

464 dependent disruption to the proteome was observed in the nuclear compartment under cell stress.

465 Since a number of prior studies have shown abnormalities in cell stress responses in DYT-

466 TOR1A models, we asked whether the nuclear proteomic disruptions were predominantly

467 composed of proteins whose levels were normally modulated by cell stress. To address this, we

468 first used the WT datasets to identify the normal subset of stress-responsive proteins - i.e.

469 proteins whose levels significantly changed in response to thapsigargin cell stress. For each

470 protein and subcellular fraction of the WT samples, the ratio of levels in the Tg and Veh

471 conditions were calculated. Using thresholds of $\pm 1.5 \mathrm{FC}$ and $\mathrm{p}$-value $<0.05$, we identified a total

472 of 513 proteins that we hereafter refer to as the "stress-responsive proteins".

Consistent with the global reduction in protein synthesis rates that occurs following ER

475 stress (Ron, 2002), Tg cell stress tended to downregulate more proteins (373 proteins) than it

476 upregulated (140 proteins) (Fig. 3A-B). Among the upregulated stress-responsive proteins in WT 
477 samples, GO analysis revealed significant enrichment for proteins associated with the PERK-

478 mediated unfolded protein response $(p=0.002$, Fold Enrichment $=10.8)$. This enrichment is

479 expected given that thapsigargin is thought to promote cellular stress response through a PERK-

480 dependent mechanism (H. P. Harding et al., 2000). Individual examples of two proteins

481 associated with this pathway, Herpud1 and NFкB p105, are shown (Fig. 3C-D).

482

483 Lastly, in reviewing the stress-responsive proteins we identified in WT samples, we were

484 surprised to see the paralog of TorsinA, TorsinB, among them. TorsinB levels were significantly

485 increased by stress in the nuclear fractions and decreased in the cytosolic fractions, resulting in a

486 greater than doubling of its relative nucleocytoplasmic distribution (nuclear:cytosolic protein

487 levels $=\mathrm{N} / \mathrm{C}$ ratio) (Fig. 3E). A similar trend was seen for TorsinA (Fig. 3F). This finding

488 suggests for the first time that redistribution of Torsin proteins toward the nucleus may be part of

489 the normal cellular stress response. 

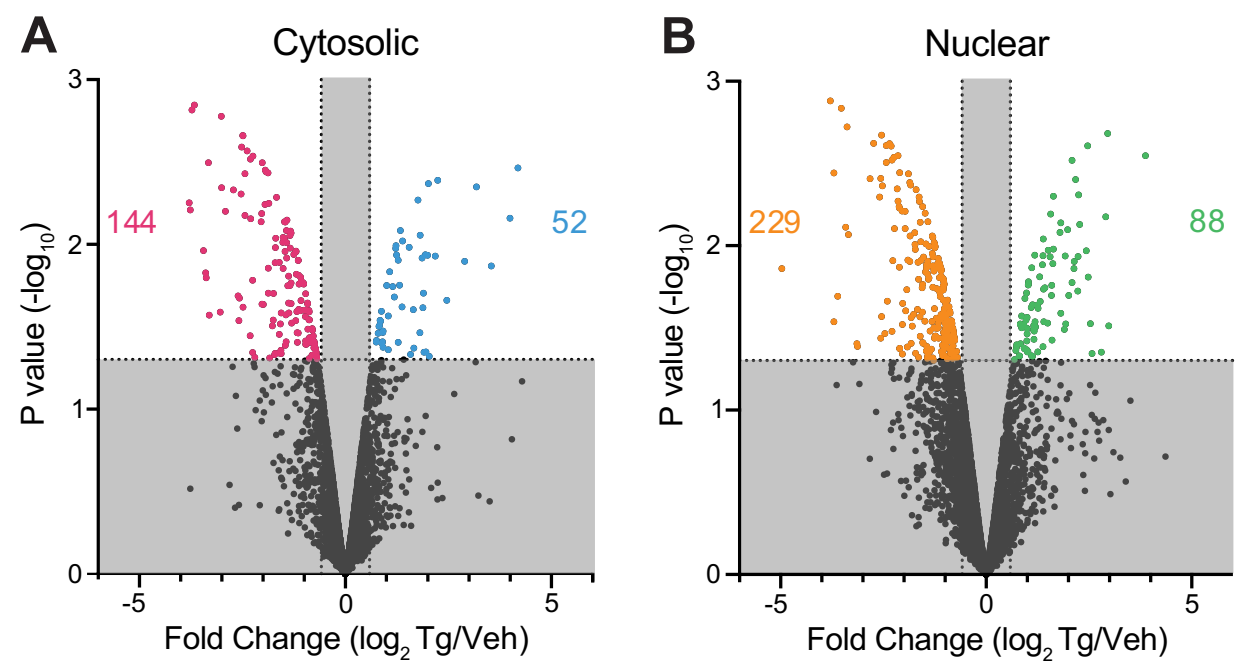

WT Tg Upregulated: • Cyto • Nuc WT Tg Downregulated: • Cyto • Nuc

$\begin{array}{llllllll}\text { C Herpud1 } & \text { D } & \text { NFkB p105 } & \text { E } & \text { TorsinB } & \text { F } & \text { TorsinA }\end{array}$
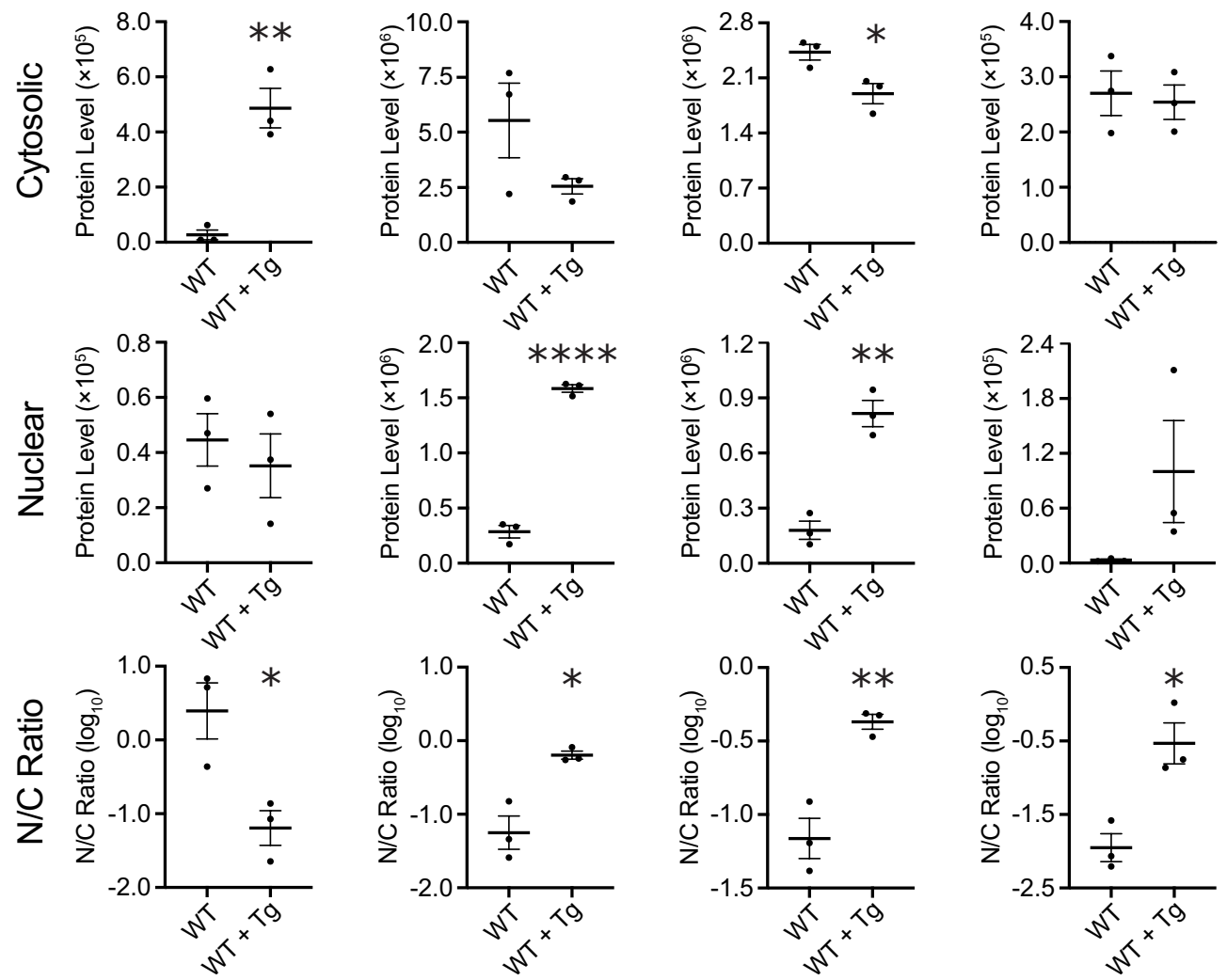
Fig. 3. Thapsigargin stress modulation of protein levels in wildtype MEFs. (A-B) Volcano plots

493 showing proteome-wide differences in protein abundances due to thapsigargin (Tg) treatment in

494 wildtype cytosolic (A) and nuclear (B) fractions. Differences in protein abundance are

495 represented as fold change (using $\log _{2}$ transform) and p-value is calculated by unpaired t-test for 496 each protein ( $\mathrm{n}=3$ biological replicates). Horizontal dashed line indicates $\mathrm{p}$-value less than 0.05.

497 Vertical dashed lines indicate fold changes of \pm 1.5 fold change (FC) (C-F) Cytosolic and nuclear 498 fraction protein levels (a.u.) and the corresponding nuclear:cytosolic (N/C) ratios are shown for

499 Herpud1, NFкB p105, TorsinB, and TorsinA in Veh (WT) and Tg treatment (WT + Tg)

500 conditions $\left(\mathrm{n}=3\right.$ biological replicates; ${ }^{*} \mathrm{p}<0.05 ; * \mathrm{*}<0.01 ; * * * \mathrm{p}<0.0001$, unpaired t-test). Error 501 bars indicate S.E.M..

\section{DYT-TOR1A MEFs Show Basal Elevations and Blunted Stress Responses of}

\section{Normally Stress-Responsive Proteins}

Having defined the normal stress-responsive shifts in the proteome, we next evaluated

whether the WT stress-responsive proteins were enriched among DYT-TOR1A disrupted

507 proteins. We found that stress-responsive proteins were significantly enriched among the DYT-

508 TOR1A disrupted proteins. This enrichment was present across all conditions and subcellular

509 fractions. To visualize this, in Fig. 4 we show the DYT-TOR1A/WT comparison datasets using

510 the color scheme from Fig. 3 to indicate the nature of the WT stress response (i.e. upregulated or

511 downregulated in Fig. 3A-B). Notably, under basal conditions (Veh), WT stress-responsive

512 proteins were not uniformly distributed but rather tended to align with the directionality of their

513 normal modulation by cell stress. As a group, the proteins upregulated by thapsigargin within

514 WT MEFs were significantly enriched among the subset of proteins upregulated in DYT-TOR1A 
515 basally (Cyto: $p=7.68 \mathrm{e}-8$; Nuc: $p=5.98 \mathrm{e}-12$ ) (Fig. 4A-B). Similarly, the group of proteins

516 downregulated by Tg treatment in WT MEFs were significantly enriched among the subset of

517 proteins downregulated in DYT-TOR1A basally (Cyto: $p=6.92 \mathrm{e}-6$; Nuc: $\mathrm{p}=8.81 \mathrm{e}-11$ ) (Fig. 4A-

518 B). This analysis reveals that the basal state proteome of DYT-TOR1A MEFs reflects an

519 activated cell stress state prior to exogenous Tg cell stress treatment.

Following Tg stress treatment, we again found significant overlap between the normal

522 (WT) stress-responsive proteins and DYT-TOR1A genotype-dependent protein disruptions in

523 both subcellular compartments (Fig. 4C-D). However, under Tg cell stress, the directionality of

524 the genotype-dependent disruptions was opposite to that of the normal stress response (visualized

525 by the colored symbols concentrating on the opposite side of the volcano plots, Fig. 4C-D).

526 Upregulated WT stress-responsive proteins were significantly enriched among the subset of

527 downregulated proteins in Tg-treated DYT-TOR1A fractions when compared to Tg-treated WT

528 fractions (Cyto: $\mathrm{p}=4.38 \mathrm{e}-12$; Nuc: p=9.92e-3) (Fig. 4C-D). Likewise, downregulated WT stress-

529 responsive proteins were significantly enriched among the subset of upregulated proteins in $\mathrm{Tg}$ -

530 treated DYT-TOR1A fractions compared to Tg-treated WT fractions (Cyto: $\mathrm{p}=4.67 \mathrm{e}-13$; Nuc:

$531 \mathrm{p}=2.09 \mathrm{e}-12)$ (Fig. 4C-D). These findings are consistent with a blunted stress response in DYT-

532 TOR1A MEFs following Tg cell stress.

At the level of individual proteins, we noticed that these same trends could be seen in the

$535 \mathrm{~N} / \mathrm{C}$ ratios of $\mathrm{NF \kappa B}$ p105 and TorsinA, but also, that for others, stress-regulation was unaffected

536 by the DYT-TOR1A genotype (e.g. Herpud1 and TorsinB) (Fig. 5A-D, S3). Given this variation,

537 we sought to quantify the average degree of modulation across the entire population of WT 
538 stress-responsive proteins. We measured the fold change for each of the 140 WT stress-

539 upregulated proteins and 373 WT stress-downregulated proteins in each condition relative to its

540 level in the basal state WT samples (Veh), and then calculated the mean fold change for all up-

541 or downregulated proteins in each condition (Fig. 5E-F). This analysis shows that in the basal

542 state, levels of WT stress-responsive proteins in DYT-TOR1A samples already have modulations

543 in the direction consistent with stress responses - the mean level of WT stress-upregulated

544 proteins was $\sim 60$ percent $(\mathrm{p}=8.63 \mathrm{e}-19)$ higher than WT basal levels, while the mean level of

545 WT stress-downregulated proteins was $\sim 15$ percent lower $(\mathrm{p}=1.95 \mathrm{e}-5)$. These findings indicate

546 that the nuclear and cytosolic proteomes in DYT-TOR1A MEFs show modulations consistent

547 with a stressed state basally.

In WT cells, cell stress by Tg exposure caused a threefold change in the mean level of 550 upregulated and downregulated WT stress-responsive proteins (Fig. 5E-F). By comparison, in 551 DYT-TOR1A MEFs, Tg caused only a twofold change in the levels of these same WT stress552 responsive proteins (upregulated: $p=8.63 \mathrm{e}-19$; downregulated: $p=3.40 \mathrm{e}-16$ ) (Fig. 5E-F). These

553 results indicate that while the DYT-TOR1A proteome does respond to cellular stress, the 554 magnitude of the response is significantly reduced. 

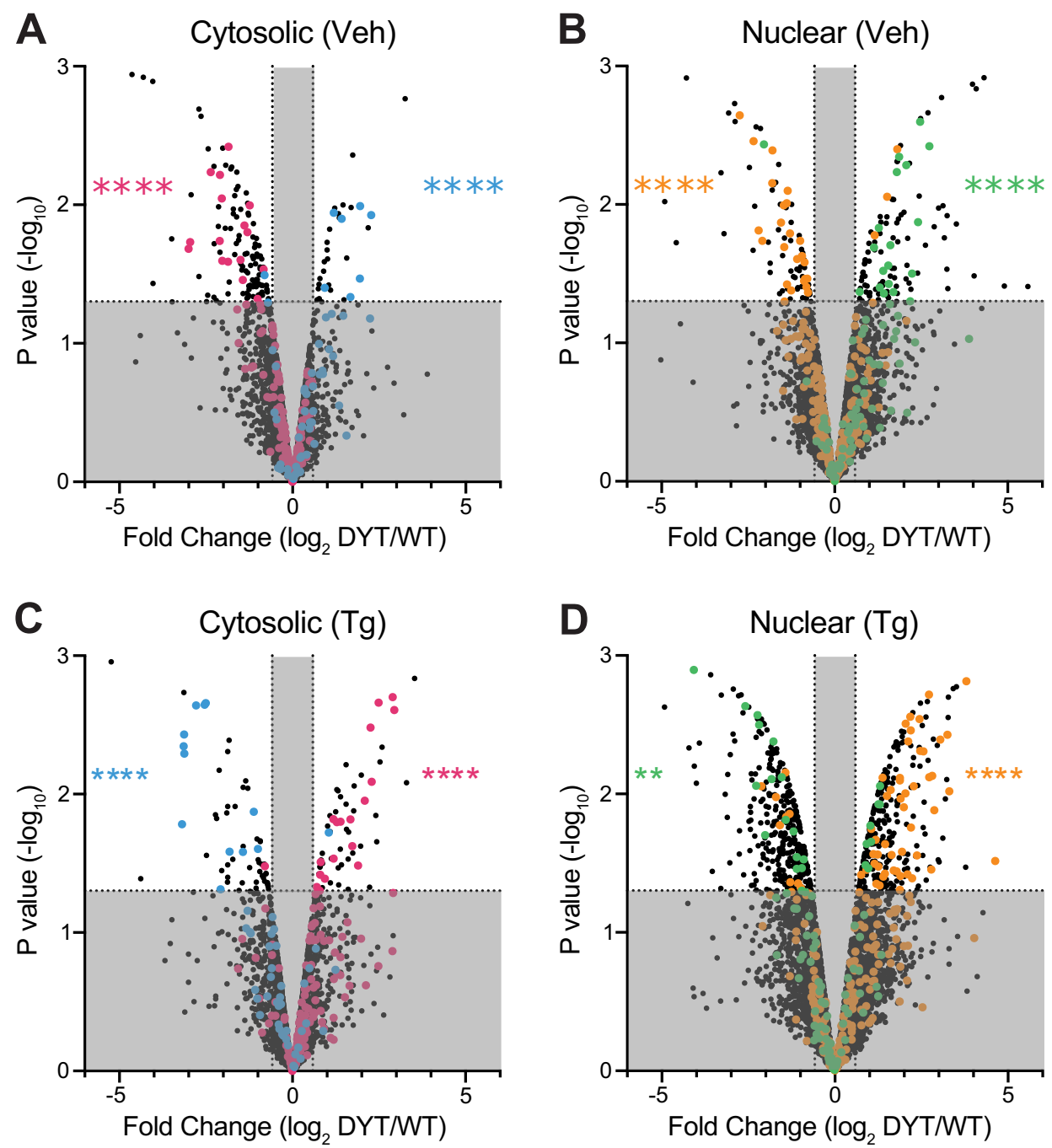

WT Tg Upregulated: • Cyto • Nuc WT Tg Downregulated: • Cyto • Nuc

557 Fig. 4. WT Tg-stress-responsive proteins are non-randomly distributed among DYT-TOR1A

558 genotype-dependent proteome disruptions. Volcano plots showing proteome-wide differences in

559 protein abundances between DYT-TOR1A and WT cytosolic and nuclear fractions under basal

560 (A, B) and thapsigargin-treated (C, D) conditions. For proteins found to be Tg-modulated in WT

561 samples (Fig. 3), the directionality of the WT sample modulation is indicated by symbol color

562 (see Legend). Differences in protein abundance are represented as fold change (using $\log _{2}$

563 transform) and $\mathrm{p}$-value is calculated by unpaired t-test for each protein $(\mathrm{n}=3$ biological 
564 replicates). Horizontal dashed line indicates $p$-value less than 0.05 . Vertical dashed lines indicate

565 fold changes of \pm 1.5 FC. Asterisks indicate p-value calculated by Fisher's exact test for overlap

566 between the indicated subsets of DYT1 disrupted proteins and WT Tg-stress-responsive proteins

567 in the white quadrants $(* * \mathrm{p}<0.01 ; * * * * \mathrm{p}<0.0001)$. 

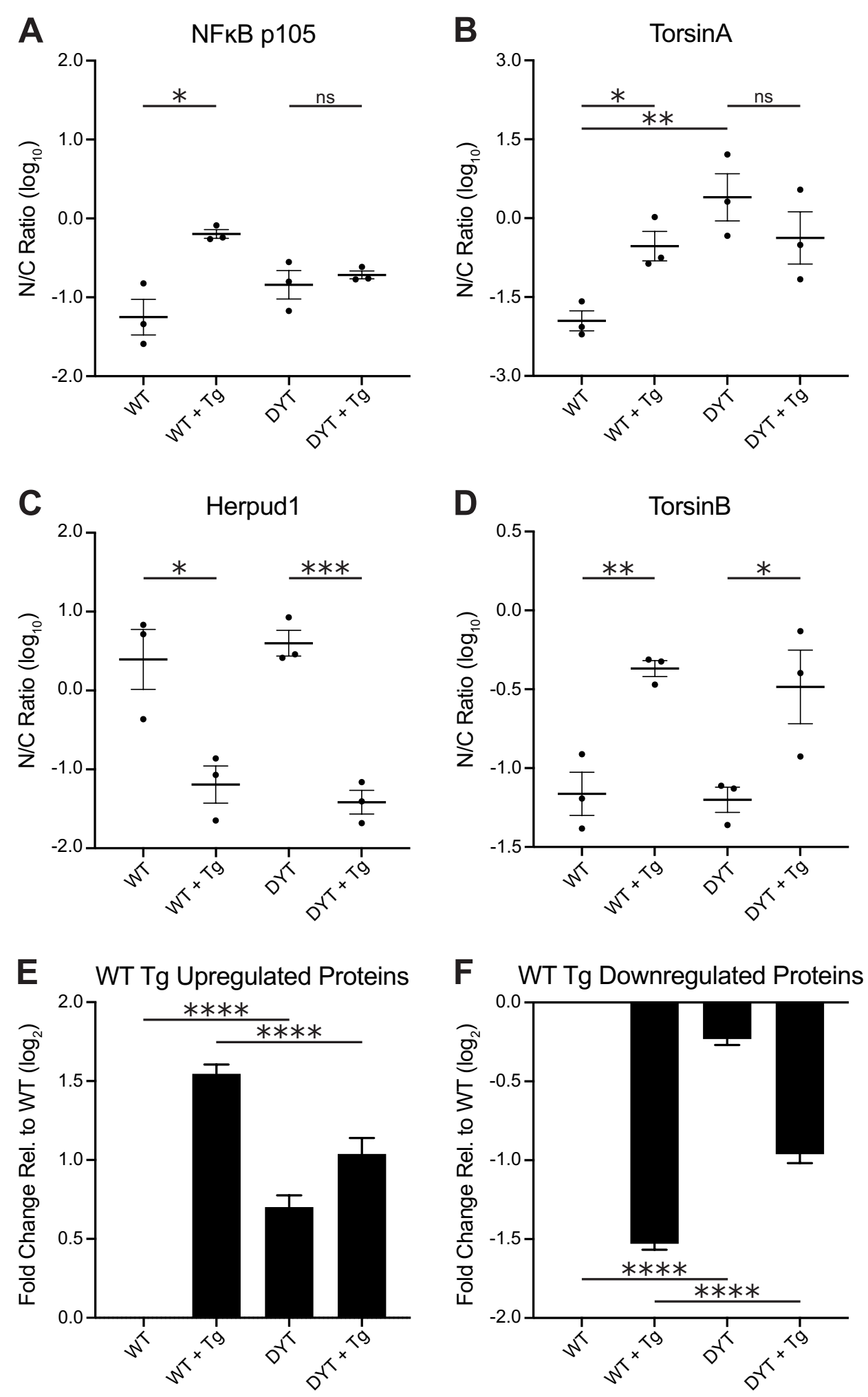
Fig. 5. DYT-TOR1A MEFs show proteome-wide disruptions consistent with an elevated basalstate stress response and a blunted response to Tg stress. (A-D) Individual protein examples of

572 Tg stress-induced shifts in protein abundance between nuclear and cytosolic fractions are shown

573 for NFkB p105 (A), TorsinA (B), Herpud1 (C), and TorsinB (D) in WT and DYT-TOR1A MEFs

574 ( $\mathrm{n}=3$ biological replicates) $\left({ }^{*} \mathrm{p}<0.05 ; * * \mathrm{p}<0.01 ; * * * \mathrm{p}<0.001\right.$, unpaired t-test). (E-F) Mean fold

575 change calculated across all WT Tg-stress-responsive upregulated $(n=140)(E)$ and

576 downregulated (n=373) (F) proteins. For this calculation, protein levels (a.u.) from WT and

577 DYT-TOR1A fractions treated with either Tg or Veh were normalized to the protein level

578 measured in the WT Veh-treated fraction from the same subcellular compartment $(\mathrm{n}=3 \mathrm{FC}$

579 values per protein to calculate mean value). Significance was determined by unpaired t-test

580 between mean $\mathrm{FC}$ values for 140 upregulated stress-responsive proteins $(\mathrm{E})(* * * * \mathrm{p}<0.0001)$ and

581373 downregulated stress-responsive proteins $(\mathrm{F})(* * * * \mathrm{p}<0.0001)$. Error bars indicate S.E.M..

583 Dysregulation of WT Stress-Responsive Proteins Does Not Explain

584 Accentuated Proteome Disruption in Stressed Nuclear DYT-TOR1A

\section{Fractions}

Thus far, we have found that WT stress-responsive proteins are significantly enriched

587 among the proteins whose levels are disrupted by DYT-TOR1A (Fig. 4). Because this

588 enrichment is similarly observed in both subcellular compartments and under both basal and $\mathrm{Tg}$

589 cell stress (Fig. 4), it cannot explain the large, Tg and nucleus-selective disruption of 624

590 proteins caused by DYT-TOR1A. Moreover, stress-responsive proteins explain only $13 \%$ of the

591 total number of disrupted proteins in the Tg nuclear samples (Fig. 6A). We therefore sought to

592 further understand the nature of the proteins disrupted by DYT-TOR1A in the nucleus under 
593 stress (Fig. 6A-C). A GO analysis was performed on the set of 624 proteins differentially

594 regulated in DYT-TOR1A + Tg nuclear fractions as compared to the WT + Tg nuclear fractions

595 (Fig. 6D, Table S1). Enrichments included "neuron cellular homeostasis" (p=0.002, Fold

596 Enrichment=4.5), "mRNA-containing ribonucleoprotein (RNP) complex export from the

597 nucleus" $(p=0.004$, Fold Enrichment $=1.8)$, and "axonal transport" $(p=0.006$, Fold

598 Enrichment=2.3) (Fig. 6D and Table S1). These GO terms suggest that in addition to the

599 pervasive disruptions in cell stress responses, the DYT-TOR1A genotype may also cause

600 particular liabilities in the nucleus under cell stress among proteins generally important for

601 synaptic and neuronal function.

602

A

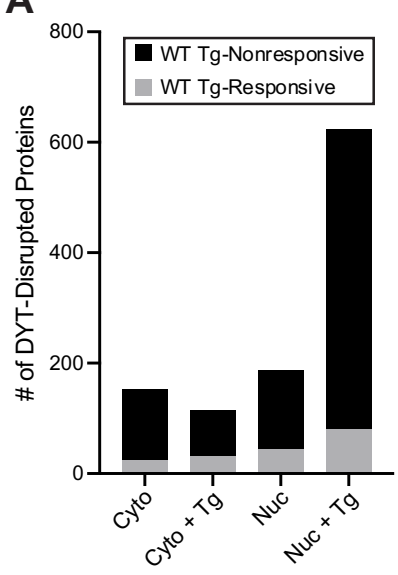

D

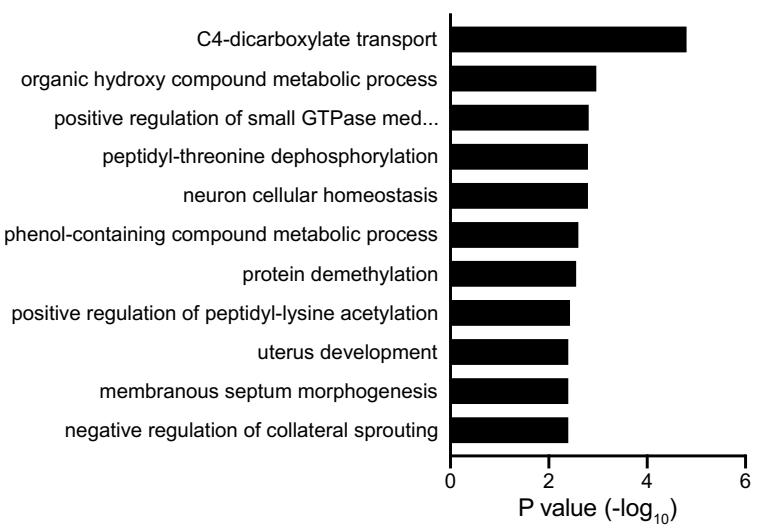

B

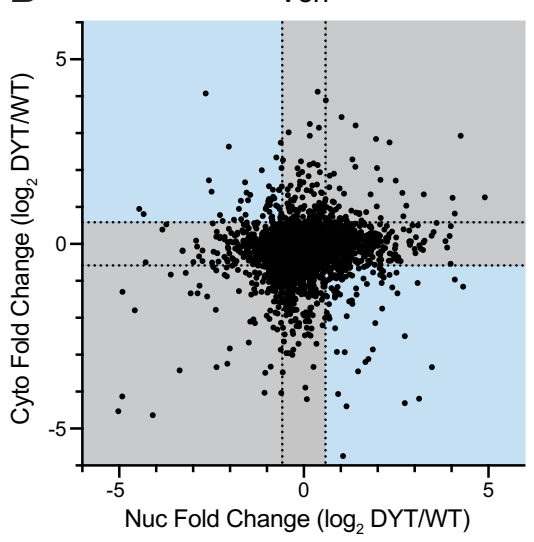

C $\mathrm{Tg}$

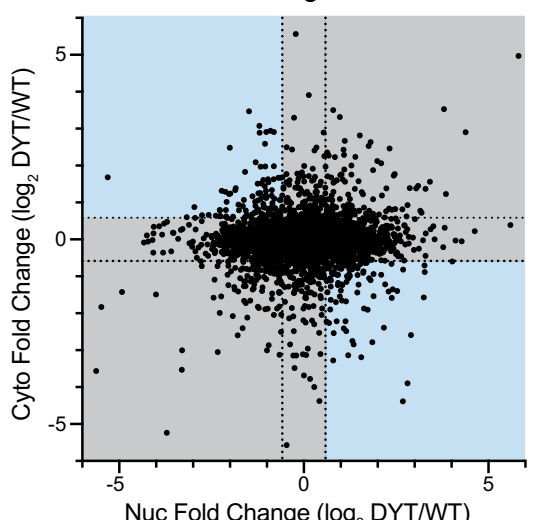

Nuc Fold Change $\left(\log _{2}\right.$ DYT/WT)

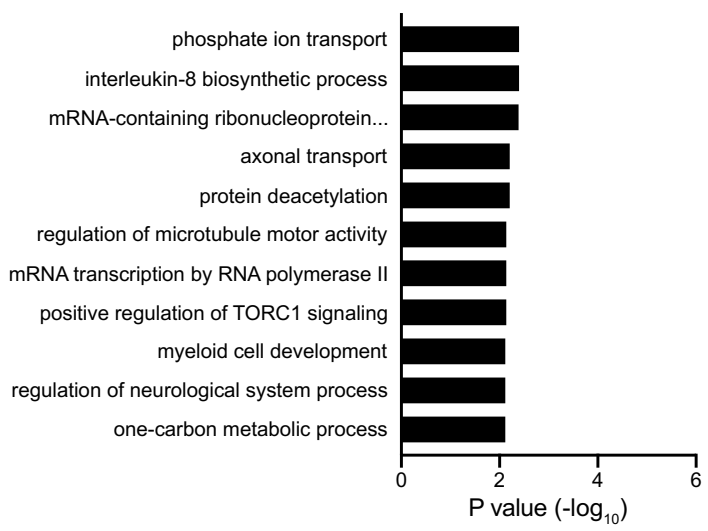


604 Fig. 6. Stress-dependent nuclear proteome disruptions in DYT-TOR1A MEFs associate with

605 critical neuronal processes. (A) Absolute number of proteins with significant genotype effects

$606(\mathrm{FC}> \pm 1.5, \mathrm{p}<0.05)$ in each cellular fraction and treatment condition. (B, C) Proteome-wide

607 results for genotype effects comparing directionality of fold change between the nuclear and

608 cytosolic compartments in the vehicle (B) and thapsigargin (C) conditions. Blue shading denotes

609 zones of reciprocal relationships. Gray shaded regions are FC less than \pm 1.5 . (D) Gene Ontology

610 analysis of the 624 proteins in the Tg-nuclear fractions with significant DYT-TOR1A genotype-

611 dependent effects $(\mathrm{FC}> \pm 1.5, \mathrm{p}<0.05)$

612

613 Discussion

In this study, we performed quantitative proteomic analysis of nuclear and cytosolic-

615 enriched fractions prepared from DYT-TOR1A and WT MEFs in order to identify cell

616 compartment-specific disruptions. This proteomic approach was driven by our hypothesis that

617 some protein level disruptions caused by the DYT-TOR1A genotype may differentially manifest

618 between the nuclear and cytosolic compartments given associations of Torsin $\mathrm{A}$ and $\Delta \mathrm{E}$ Torsin $\mathrm{A}$

619 with nuclear envelope structure and nuclear transport functions (Chase et al., 2017; Ding et al.,

620 2021; Gonzalez-Alegre \& Paulson, 2004; Goodchild et al., 2005; Goodchild \& Dauer, 2004,

621 2005; György et al., 2018; Jokhi et al., 2013; M. T. Jungwirth et al., 2011; Laudermilch et al.,

622 2016; Naismith et al., 2004; Nery et al., 2011; Pappas et al., 2018; Rampello et al., 2019; Tanabe

623 et al., 2016; VanGompel et al., 2015). To date, proteomic studies of dystonia have examined

624 whole cell and tissue lysates (Beauvais et al., 2016; Martin et al., 2009; Zakirova et al., 2018).

625 Using nucleocytoplasmic proteomics, we discovered that the DYT-TOR1A mutation

626 disproportionately causes the nuclear proteome to be disrupted by cell stress. There were 3-fold 
627 more disrupted proteins in the thapsigargin-treated nuclear proteome than any other condition

628 (Fig. 6). Although this effect occurred under Tg cell stress and we found that levels of many

629 proteins that were normally modulated by Tg were disrupted in DYT-TOR1A, the majority of

630 the affected proteins were not part of the WT stress-responsive subset. In addition to this novel

631 finding, our datasets provide further support for several prior observations in the field. For

632 example, in both subcellular compartments, DYT-TOR1A samples show evidence of impaired

633 mitochondrial function and stress responses (Beauvais et al., 2016; Cao et al., 2005; Chen et al.,

634 2010; Martin et al., 2009; Nery et al., 2011; Rittiner et al., 2016).

To begin to address the potential impact of the stress-dependent nuclear proteome

637 disruptions in DYT-TOR1A MEFs on dystonia pathophysiology, we conducted Gene Ontology

638 analysis. Despite the source cells being non-neuronal, a number of GO terms with particular

639 importance for neuronal function were enriched (Fig. 6). These include: "Neuron Cellular

640 Homeostasis", "Negative Regulation of Collateral Sprouting”, "mRNA-containing

641 Ribonucleoprotein Complex Export from the Nucleus", “Axonal Transport”, "Regulation of

642 Microtubule Motor Activity", "Positive Regulation of TORC1 Signaling”, and "Regulation of

643 Neurological System Process". One of the identified biological functions enriched among the

644 stress-dependent nuclear proteome disruptions caused by the DYT-TOR1A genotype was

645 "mRNA-containing ribonucleoprotein complex export from the nucleus". Prior studies have

646 made an association between a particular type of RNP complex, a "megaRNP” and TorsinA

647 function (Jokhi et al., 2013). In D. melanogaster, nuclear egress of the megaRNP is essential for

648 proper synaptic development of the neuromuscular junction (Speese et al., 2012). More

649 generally, ribonucleoprotein complexes are known to play an integral role in the maintenance, 
650 stress response, and plasticity of synapses, and RNP disruptions are associated with a number of

651 neurological diseases (Khalil et al., 2018; Ross Buchan, 2014). Therefore, taken together, the GO

652 processes involving RNP complexes, transport processes, and neuronal homeostasis are

653 potentially interrelated and predict impact on critical brain processes. Our findings suggest that

654 these processes may be most disrupted in settings of cell stress - whether due to inherent states,

655 such as development, or exogenous cell stressors. An important future direction is to determine

656 whether the stress-dependent vulnerability of the nuclear proteome observed in this study also

657 exists within the mammalian central nervous system and, if so, which neural cells are most

658 affected by this vulnerability.

Our proteomic data identify protein levels that differ because of the DYT-TOR1A

661 genotype. In interpreting these data, there are a number of factors that could lead to altered

662 protein levels. Such factors include changes in protein synthesis rates, protein degradation rates,

663 transport between subcellular compartments or aggregation state. TorsinA is well known to

664 move between the lumen of the endoplasmic reticulum and the outer nuclear envelope in an

665 ATP-hydrolysis dependent manner (Goodchild \& Dauer, 2004, 2005; Naismith et al., 2004;

666 Vander Heyden et al., 2009; Zhao et al., 2013). Moreover, a number of experimental approaches

667 have shown that TorsinA deletion or $\Delta \mathrm{E}$ TorsinA overexpression disrupts this trafficking and

668 nuclear envelope structure (Ding et al., 2021; Gonzalez-Alegre \& Paulson, 2004; Goodchild et

669 al., 2015; Goodchild \& Dauer, 2004; M. T. Jungwirth et al., 2011; Naismith et al., 2004; Torres

670 et al., 2004; Vander Heyden et al., 2009). We therefore found it notable that our proteomic data

671 did not provide support for a model where $\Delta \mathrm{E}$ TorsinA restricts protein trafficking between the

672 nucleus and cytosol. More specifically, we performed an analysis across the entire detected 
673 proteome irrespective of the p-values to determine if we could detect even a trend for proteins to

674 show reciprocal relationships between nuclear and cytosolic fractions (i.e. lowering in one

675 compartment and increasing in the other) (Fig. 6B-C, blue areas). Such changes would be

676 represented in the colored quadrants of those graphs and create an elliptical skewing. However,

677 in this analysis, we saw no trends for reciprocal changes. Instead, we see that basally, there was

678 little if any skew (Fig. 6B) and that with thapsigargin, the major modulation was restricted to the

679 nuclear compartment (e.g. data expanding horizontally, nuclear fold-change axis) with little

680 change vertically (cytosolic fold-change axis) (Fig. 6C). We therefore favor models other than

681 nucleocytoplasmic transport defects to explain the bulk of proteome disruption in DYT-TOR1A

682 MEFs. As one example of alternative models, compartment-specific protein level disruptions

683 could arise by dysregulation of compartment-specific protein degradation mechanisms, such as

684 the nuclear proteasome or ER-associated degradation mechanisms (Enenkel, 2014; Nery et al.,

685 2011). We are interested in testing this possibility in future studies.

In addition to identifying nuclear compartment-specific disruptions that were largely

688 unrelated to WT stress-responsive proteins, we also made a number of novel observations about

689 the integrity of the stress response in DYT-TOR1A cells. Foremost among these, by using a

690 proteome-wide approach as opposed to monitoring a small number of proteins of interest, we

691 found that, as a group, proteins whose levels were normally modulated by cell stress (in this

692 experiment, by thapsigargin) tended to show deviations toward their stress response in the basal

693 state in DYT-TOR1A cells (Fig. 4,5E-F). This result suggests that the DYT-TOR1A genotype

694 induces a basally elevated stress state. 

cellular models. For example, BiP, a key stress-responsive protein, is upregulated in an

698 unstressed C. elegans DYT-TOR1A transgenic model (Chen et al., 2010). In this study, the

699 investigators further noted that in response to cell stress (using tunicamycin, a glycosylation

700 inhibitor), DYT-TOR1A model worms had an exaggerated BiP response. Tunicamycin increased

701 BiP expression in both WT and DYT-TOR1A samples, but to a greater degree in DYT-TOR1A.

702 To explain the elevated stress response both basally and following stress, the authors speculated

703 that DYT-TOR1A cells have a reduced buffering capacity against cell stress. In our data, we

704 confirm the observations of Chen and colleagues - levels of BiP are greater in DYT-TOR1A

705 than WT basally and increase more in DYT-TOR1A than WT in response to thapsigargin (Fig.

706 S7B). However, by looking at the entire set of experimentally identified stress-responsive

707 proteins, we further recognized that the BiP response was not representative of the average

708 response to cell stress in DYT-TOR1A MEFs. Instead, we find that the DYT-TOR1A genotype

709 lowers the overall stress response relative to WT (Fig. 5E-F). Blunting of the stress response has

710 also been reported in primary fibroblasts from DYT-TOR1A patients and cerebellar tissue from

711 DYT-TOR1A mouse models (Beauvais et al., 2016; Rittiner et al., 2016). Based on our new

712 findings, we postulate that homeostatic dysregulation of stress signaling pathways may have

713 developed due to the chronically elevated stress response in DYT-TOR1A cells that exists prior

714 to exogenous stress treatment. An important area for future studies in DYT-TOR1A is to identify

715 the biological mechanisms inciting basal cell stress and driving homeostatic dysregulation.

Although our proteomic experiments were not designed to address single protein-level

718 hypotheses, our results make three preliminary novel observations regarding Torsins. First, to 
719 our knowledge, we make the first observation that TorsinB is a stress-responsive protein.

720 Second, levels of TorsinA also appear to be stress-modulated. However, in contrast to TorsinB,

721 TorsinA stress modulation is impaired (occluded) in DYT-TOR1A cells, while the response of

722 TorsinB appears normal (Fig. 5B, D). This difference between TorsinA and TorsinB is

723 noteworthy because a number of prior studies have highlighted the potential for TorsinB to

724 substitute for loss of TorsinA function in DYT-TOR1A (M. Jungwirth et al., 2010; Tanabe et al.,

725 2016; Vasudevan et al., 2006), including a recent study which shows that overexpression of

726 TorsinB rescues abnormal movement phenotypes observed in forebrain specific Torla and

727 Torla/Torlb combined conditional knockout as well as selective Tor $1 a^{\Delta \mathrm{GAG}}$ knock-in mouse

728 models (Li et al., 2020). Our data provide additional support for the rationale of such therapeutic

729 approaches. Third and last, with the sensitivity afforded by proteomic methodologies, we find

730 that TorsinA is mislocalized toward the nuclear compartment in DYT-TOR1A cells with genetic

731 construct validity. Although mislocalization of $\Delta \mathrm{E}$ Torsin A has been widely observed across labs

732 and experimental settings (Bragg et al., 2004; Calakos et al., 2010; Gonzalez-Alegre \& Paulson,

733 2004; Goodchild \& Dauer, 2004; Hewett et al., 2000; Kustedjo et al., 2000; Liang et al., 2014;

734 Naismith et al., 2004; Torres et al., 2004), to our knowledge it has never been documented in a

735 construct-valid genetic model for DYT-TOR1A dystonia (e.g. Tor $1 a^{\Delta \mathrm{GAG} /+}$ ). Our results

736 therefore provide experimental support for the idea that $\Delta \mathrm{E}$ TorsinA mislocalization exists in the

737 genetically relevant setting and it may only be the matter of degree that differs from

738 overexpression models. 


\section{Conclusions}

743 We have newly identified a stress-dependent and nuclear compartment-specific proteome

744 disruption caused by the DYT-TOR1A genotype. Our findings suggest that key brain processes

745 involving neuronal homeostasis, transport, and RNP export may be selectively impaired in DYT-

746 TOR1A by cell stress through effects on the nuclear proteome. Alongside further understanding

747 TorsinA's role in modifying cellular stress responses, our results open a new research direction

748 for DYT-TOR1A dystonia pathophysiology which is to understand this compartment-specific

749 vulnerability and its consequences for brain function.

750

751 
Author Contributions:

753

754 KS - Conceptualization, Investigation, Formal analysis, Writing - Original Draft, Review \&

755 Editing

756 ZFC -Supervision, Validation, Writing - Review \& Editing

757 NC - Conceptualization, Funding acquisition, Supervision, Writing - Review \& Editing

Acknowledgements:

The authors wish to acknowledge critical expertise and suggestions provided by Shataakshi Proteomics and Metabolomics Core. K.S. thanks the members of his undergraduate thesis

Funding:

772 Huang Undergraduate Summer Research Fellowship (K.S.), Duke Health Scholar award (N.C.), 773 Cure Dystonia Now (N.C.), Dystonia Medical Research Foundation (N.C.) and Tyler's Hope for 774 a Dystonia Cure (N.C.).

780 The authors have no competing financial interest. 
782

783

784

785

786

787

788

789

790

791

792

793

794

795

796

797

798

799

800

801

802

803

804

\section{References}

Balint, B., Mencacci, N. E., Valente, E. M., Pisani, A., Rothwell, J., Jankovic, J., Vidailhet, M., \& Bhatia, K. P. (2018). Dystonia. Nature Reviews Disease Primers, 4(1), 1-23. https://doi.org/10.1038/s41572-018-0023-6

Beauvais, G., Bode, N. M., Watson, J. L., Wen, H., Glenn, K. A., Kawano, H., Charles Harata, X. N., Ehrlich, M. E., \& Gonzalez-Alegre, P. (2016). Disruption of Protein Processing in the Endoplasmic Reticulum of DYT1 Knock-in Mice Implicates Novel Pathways in Dystonia Pathogenesis. https://doi.org/10.1523/JNEUROSCI.0669-16.2016

Beauvais, G., Rodriguez-Losada, N., Ying, L., Zakirova, Z., Watson, J. L., Readhead, B., Gadue, P., French, D. L., Ehrlich, M. E., \& Gonzalez-Alegre, P. (2018). Exploring the Interaction Between eIF2 $\alpha$ Dysregulation, Acute Endoplasmic Reticulum Stress and DYT1 Dystonia in the Mammalian Brain. Neuroscience, 371, 455-468.

https://doi.org/10.1016/j.neuroscience.2017.12.033

Bragg, D. C., Kaufman, C. A., Kock, N., \& Breakefield, X. O. (2004). Inhibition of N-linked glycosylation prevents inclusion formation by the dystonia-related mutant form of torsinA. Molecular and Cellular Neuroscience, 27(4), 417-426. https://doi.org/10.1016/j.mcn.2004.07.009

Bressman, S. B. (2004). Dystonia genotypes, phenotypes, and classification. In Advances in neurology.

Calakos, N., Patel, V. D., Gottron, M., Wang, G., Tran-Viet, K. N., Brewington, D., Beyer, J. L., Steffens, D. C., Krishnan, R. R., \& Züchner, S. (2010). Functional evidence implicating a novel TOR1A mutation in idiopathic, late-onset focal dystonia. Journal of Medical Genetics, 47(9), 646-650. https://doi.org/10.1136/jmg.2009.072082 
Cao, S., Gelwix, C. C., Caldwell, K. A., \& Caldwell, G. A. (2005). Torsin-mediated protection from cellular stress in the dopaminergic neurons of Caenorhabditis elegans. Journal of Neuroscience, 25(15), 3801-3812. https://doi.org/10.1523/JNEUROSCI.5157-04.2005

808 Carr, S., Aebersold, R., Baldwin, M., Burlingame, A., Clauser, K., \& Nesvizhskii, A. (2004). The need for guidelines in publication of peptide and protein identification data: Working group on publication guidelines for peptide and protein identification data. In Molecular and Cellular Proteomics (Vol. 3, Issue 6, pp. 531-532). American Society for Biochemistry and Molecular Biology. https://doi.org/10.1074/mcp.T400006-MCP200 in a heterologous yeast system reveals interactions with lumenal domains of LINC and nuclear pore complex components. Molecular Biology of the Cell, 30(5), 530-541.

Chen, P., Burdette, A. J., Porter, J. C., Ricketts, J. C., Fox, S. A., Nery, F. C., Hewett, J. W., Dynamic functional assembly of the Torsin AAA+ ATPase and its modulation by LAP1. Molecular Biology of the Cell. https://doi.org/10.1091/mbc.E17-05-0281 Berkowitz, L. A., Breakefield, X. O., Caldwell, K. A., \& Caldwell, G. A. (2010). The early- 
Ding, B., Tang, Y., Ma, S., Akter, M., Liu, M. L., Zang, T., \& Zhang, C. L. (2021). Disease modeling with human neurons reveals lmnb1 dysregulation underlying dyt1 dystonia. Journal of Neuroscience. https://doi.org/10.1523/JNEUROSCI.2507-20.2020

Enenkel, C. (2014). Nuclear transport of yeast proteasomes. In Biomolecules (Vol. 4, Issue 4, pp. 940-955). MDPI AG. https://doi.org/10.3390/biom4040940

Esra Demircioglu, F., Sosa, B. A., Ingram, J., Ploegh, H. L., \& Schwartz, T. U. (2016). Structures of torsinA and its disease-mutant complexed with an activator reveal the molecular basis for primary dystonia. ELife. https://doi.org/10.7554/eLife.17983

Gonzalez-Alegre, P., \& Paulson, H. L. (2004). Aberrant Cellular Behavior of Mutant TorsinA Implicates Nuclear Envelope Dysfunction in DYT1 Dystonia. Journal of Neuroscience, 24(11), 2593-2601. https://doi.org/10.1523/JNEUROSCI.4461-03.2004

Goodchild, R. E., Buchwalter, A. L., Naismith, T. V., Holbrook, K., Billion, K., Dauer, W. T., Liang, C. C., Dear, M. L., \& Hanson, P. I. (2015). Access of torsinA to the inner nuclear membrane is activity dependent and regulated in the endoplasmic reticulum. Journal of Cell Science, 128(15), 2854-2865. https://doi.org/10.1242/jcs.167452

Goodchild, R. E., \& Dauer, W. T. (2004). Mislocalization to the nuclear envelope: An effect of the dystonia-causing torsinA mutation. Proceedings of the National Academy of Sciences of the United States of America, 101(3), 847-852. https://doi.org/10.1073/pnas.0304375101

Goodchild, R. E., \& Dauer, W. T. (2005). The AAA+ protein torsinA interacts with a conserved domain present in LAP1 and a novel ER protein. Journal of Cell Biology, 168(6), 855-862. https://doi.org/10.1083/jcb.200411026

Goodchild, R. E., Kim, C. E., \& Dauer, W. T. (2005). Loss of the dystonia-associated protein torsinA selectively disrupts the neuronal nuclear envelope. Neuron, 48(6), 923-932. 
https://doi.org/10.1016/j.neuron.2005.11.010

852

853
György, B., Cruz, L., Yellen, D., Aufiero, M., Alland, I., Zhang, X., Ericsson, M., Fraefel, C., Li, Y. C., Takeda, S., Hyman, B. T., \& Breakefield, X. O. (2018). Mutant torsinA in the heterozygous DYT1 state compromises HSV propagation in infected neurons and fibroblasts. Scientific Reports, 8(1). https://doi.org/10.1038/s41598-018-19865-2

Harding, H. (2003). Immortalization of MEF with SV40 T antigen. In Internet.

Harding, H. P., Zhang, Y., Bertolotti, A., Zeng, H., \& Ron, D. (2000). Perk is essential for translational regulation and cell survival during the unfolded protein response. Molecular Cell, 5(5), 897-904. https://doi.org/10.1016/S1097-2765(00)80330-5

Hewett, J., Gonzalez-Agosti, C., Slater, D., Ziefer, P., Li, S., Bergeron, D., Jacoby, D. J., Ozelius, L. J., Ramesh, V., \& Breakefield, X. O. (2000). Mutant torsinA, responsible for early-onset torsion dystonia, forms membrane inclusions in cultured neural cells. Human Molecular Genetics. https://doi.org/10.1093/hmg/9.9.1403

Jankovic, J., \& Tintner, R. (2001). Dystonia and parkinsonism. Parkinsonism and Related Disorders, 8(2), 109-121. https://doi.org/10.1016/S1353-8020(01)00025-6

Jokhi, V., Ashley, J., Nunnari, J., Noma, A., Ito, N., Wakabayashi-Ito, N., Moore, M. J., \& Budnik, V. (2013). Torsin Mediates Primary Envelopment of Large Ribonucleoprotein Granules at the Nuclear Envelope. Cell Reports. https://doi.org/10.1016/j.celrep.2013.03.015

Jozefczuk, J., Drews, K., \& Adjaye, J. (2012). Preparation of mouse embryonic fibroblast cells suitable for culturing human embryonic and induced pluripotent stem cells. Journal of Visualized Experiments. https://doi.org/10.3791/3854

Jungwirth, M., Dear, M. L., Brown, P., Holbrook, K., \& Goodchild, R. (2010). Relative tissue 
expression of homologous torsinB correlates with the neuronal specific importance of

877 Jungwirth, M. T., Kumar, D., Jeong, D. Y., \& Goodchild, R. E. (2011). The nuclear envelope localization of DYT1 dystonia torsin A- $\Delta \mathrm{E}$ requires the SUN1 LINC complex component. BMC Cell Biology, 12. https://doi.org/10.1186/1471-2121-12-24

Khalil, B., Morderer, D., Price, P. L., Liu, F., \& Rossoll, W. (2018). mRNP assembly, axonal transport, and local translation in neurodegenerative diseases. In Brain Research (Vol.

Kim, C. E., Perez, A., Perkins, G., Ellisman, M. H., \& Dauer, W. T. (2010). A molecular

Kim, J. E., Hong, Y. H., Kim, J. Y., Jeon, G. S., Jung, J. H., Yoon, B. N., Son, S. Y., Lee, K. W., Kim, J. Il, \& Sung, J. J. (2017). Altered nucleocytoplasmic proteome and transcriptome distributions in an in vitro model of amyotrophic lateral sclerosis. PLoS ONE, 12(4).

Kustedjo, K., Bracey, M. H., \& Cravatt, B. F. (2000). Torsin A and its Torsion DystoniaAssociated Mutant Form Are Lumenal Glycoproteins that Exhibit Distinct Subcellular

894 Laudermilch, E., Tsai, P. L., Graham, M., Turner, E., Zhao, C., \& Schlieker, C. (2016). https://doi.org/10.1371/journal.pone.0176462 
Li, J., Liang, C. C., Pappas, S. S., \& Dauer, W. T. (2020). TorsinB overexpression prevents abnormal twisting in DYT1 dystonia mouse models. ELife, 9. https://doi.org/10.7554/eLife.54285

Liang, C. C., Tanabe, L. M., Jou, S., Chi, F., \& Dauer, W. T. (2014). TorsinA hypofunction causes abnormal twisting movements and sensorimotor circuit neurodegeneration. Journal

Martin, J. N., Bair, T. B., Bode, N., Dauer, W. T., \& Gonzalez-Alegre, P. (2009). Transcriptional and proteomic profiling in a cellular model of DYT1 dystonia. Neuroscience, 164(2), 563-

McQuin, C., Goodman, A., Chernyshev, V., Kamentsky, L., Cimini, B. A., Karhohs, K. W., Doan, M., Ding, L., Rafelski, S. M., Thirstrup, D., Wiegraebe, W., Singh, S., Becker, T., Caicedo, J. C., \& Carpenter, A. E. (2018). CellProfiler 3.0: Next-generation image processing for biology. PLoS Biology. https://doi.org/10.1371/journal.pbio.2005970 Puigserver, P., Carlsson, E., Ridderstråle, M., Laurila, E., Houstis, N., Daly, M. J., Patterson, N., Mesirov, J. P., Golub, T. R., Tamayo, P., Spiegelman, B., Lander, E. S.,

Naismith, T. V., Heuser, J. E., Breakefield, X. O., \& Hanson, P. I. (2004). TorsinA in the nuclear Hirschhorn, J. N., ... Groop, L. C. (2003). PGC-1 $\alpha$-responsive genes involved in oxidative phosphorylation are coordinately downregulated in human diabetes. Nature Genetics. https://doi.org/10.1038/ng1180 
Q., Tagaya, M., Klein, C., Tannous, B., Caldwell, K. A., Caldwell, G. A., Lencer, W. I., Ye, Y., \& Breakefield, X. O. (2011). TorsinA participates in endoplasmic reticulum-associated degradation. Nature Communications, 2(1). https://doi.org/10.1038/ncomms 1383

923 Nery, F. C., Zeng, J., Niland, B. P., Hewett, J., Farley, J., Irimia, D., Li, Y., Wiche, G.,

924 Sonnenberg, A., \& Breakefield, X. O. (2008). TorsinA binds the KASH domain of nesprins

925 and participates in linkage between nuclear envelope and cytoskeleton. Journal of Cell

927 Ortega, J. A., Daley, E. L., Kour, S., Samani, M., Tellez, L., Smith, H. S., Hall, E. A., Esengul, Y. T., Tsai, Y. H., Gendron, T. F., Donnelly, C. J., Siddique, T., Savas, J. N., Pandey, U. B., \& Kiskinis, E. (2020). Nucleocytoplasmic Proteomic Analysis Uncovers eRF1 and

Ozelius, L. J., Hewett, J. W., Page, C. E., Bressman, S. B., Kramer, P. L., Shalish, C., De Leon, Nonsense-Mediated Decay as Modifiers of ALS/FTD C9orf72 Toxicity. Neuron, 106(1), 90-107.e13. https://doi.org/10.1016/j.neuron.2020.01.020

Rampello, A. J., Laudermilch, E., Vishnoi, N., Prohet, S. M., Zhao, C., Patrick Lusk, C., \& Schlieker, C. (2019). Torsin ATPases are required to complete nuclear pore complex biogenesis in interphase 1 2. https://doi.org/10.1101/821835

942 Rittiner, J. E., Caffall, Z. F., Hernández-Martinez, R., Sanderson, S. M., Pearson, J. L., 
Tsukayama, K. K., Liu, A. Y., Xiao, C., Tracy, S., Shipman, M. K., Hickey, P., Johnson, J., Sporadic Disease Identify Impaired eIF2 $\alpha$ Signaling as a Generalizable Mechanism for Dystonia. Neuron, 92(6), 1238-1251. https://doi.org/10.1016/j.neuron.2016.11.012

948 Ron, D. (2002). Translational control in the endoplasmic reticulum stress response. Journal of Clinical Investigation, 110(10), 1383-1388. https://doi.org/10.1172/jci16784

Ross Buchan, J. (2014). MRNP granules Assembly, function, and connections with disease. In RNA Biology (Vol. 11, Issue 8, pp. 1019-1030). Landes Bioscience. https://doi.org/10.4161/15476286.2014.972208

Saunders, C. A., Harris, N. J., Willey, P. T., Woolums, B. M., Wang, Y., McQuown, A. J.,

Speese, S. D., Ashley, J., Jokhi, V., Nunnari, J., Barria, R., Li, Y., Ataman, B., Koon, A., Chang, set enrichment analysis: A knowledge-based approach for interpreting genome-wide expression profiles. Proceedings of the National Academy of Sciences of the United States 
of America, 102(43), 15545-15550. https://doi.org/10.1073/pnas.0506580102

967

968

969

970

971

972

973

974

975

976

977

978

979

980

981

982

983

984

985

986

987

988

Suzuki, K., Bose, P., Leong-Quong, R. Y., Fujita, D. J., \& Riabowol, K. (2010). REAP: A two minute cell fractionation method. BMC Research Notes. https://doi.org/10.1186/1756-0500$3-294$

Tanabe, L. M., Liang, C. C., \& Dauer, W. T. (2016). Neuronal Nuclear Membrane Budding Occurs during a Developmental Window Modulated by Torsin Paralogs. Cell Reports, 16(12), 3322-3333. https://doi.org/10.1016/j.celrep.2016.08.044

Tarsy, D., \& Simon, D. K. (2006). Dystonia. New England Journal of Medicine. https://doi.org/10.1056/NEJMra055549

Torres, G. E., Sweeney, A. L., Beaulieu, J. M., Shashidharan, P., \& Caron, M. G. (2004). Effect of torsinA on membrane proteins reveals a loss of function and a dominant-negative phenotype of the dystonia-associated $\Delta$ E-torsinA mutant. Proceedings of the National Academy of Sciences of the United States of America, 101(44), 15650-15655. https://doi.org/10.1073/pnas.0308088101

Tribl, F., Gerlach, M., Marcus, K., Asan, E., Tatschner, T., Arzberger, T., Meyer, H. E., Bringmann, G., \& Riederer, P. (2005). "Subcellular proteomics" of neuromelanin granules isolated from the human brain. In Molecular and Cellular Proteomics (Vol. 4, Issue 7, pp. 945-957). American Society for Biochemistry and Molecular Biology Inc. https://doi.org/10.1074/mcp.M400117-MCP200

van Harten, P. N., Hoek, H. W., \& Kahn, R. S. (1999). Fortnightly review: Acute dystonia induced by drug treatment. $B M J, 319(7210), 623-626$. https://doi.org/10.1136/bmj.319.7210.623

Vander Heyden, A. B., Naismith, T. V., Snapp, E. L., Hodzic, D., \& Hanson, P. I. (2009). 
LULL1 retargets torsinA to the nuclear envelope revealing an activity that is impaired by the DYT1 dystonia mutation. Molecular Biology of the Cell, 20(11), 2661-2672. https://doi.org/10.1091/mbc.E09-01-0094

992 VanGompel, M. J. W., Nguyen, K. C. Q., Hall, D. H., Dauer, W. T., \& Rose, L. S. (2015). A novel function for the Caenorhabditis elegans torsin OOC-5 in nucleoporin localization and nuclear import. Molecular Biology of the Cell. https://doi.org/10.1091/mbc.E14-07-1239

Vasudevan, A., Breakefield, X. O., \& Bhide, P. G. (2006). Developmental patterns of torsinA and torsinB expression. Brain Research, 1073-1074(1), 139-145.

Wühr, M., Güttler, T., Peshkin, L., McAlister, G. C., Sonnett, M., Ishihara, K., Groen, A. C., Presler, M., Erickson, B. K., Mitchison, T. J., Kirschner, M. W., \& Gygi, S. P. (2015). The Nuclear Proteome of a Vertebrate. Current Biology, 25(20), 2663-2671.

1002

Zacchi, L. F., Wu, H. C., Bell, S. L., Millen, L., Paton, A. W., Paton, J. C., Thomas, P. J., Zolkiewski, M., \& Brodsky, J. L. (2014). The bip molecular chaperone plays multiple roles https://doi.org/10.1016/j.cub.2015.08.047

1008 P., Ozelius, L. J., Blitzer, R. D., Gonzalez-Alegre, P., \& Ehrlich, M. E. (2018). Mutations in 1009 THAP1/DYT6 reveal that diverse dystonia genes disrupt similar neuronal pathways and 1010 functions. PLoS Genetics, 14(1). https://doi.org/10.1371/journal.pgen.1007169

1011 Zhao, C., Brown, R. S. H., Chase, A. R., Eisele, M. R., \& Schlieker, C. (2013). Regulation of 
Torsin ATPases by LAP1 and LULL1. Proceedings of the National Academy of Sciences of

1013 the United States of America, 110(17). https://doi.org/10.1073/pnas.1300676110

1014 Zhao, C., Brown, R. S. H., Tang, C. H. A., Hu, C. C. A., \& Schlieker, C. (2016). Site-specific

1015 proteolysis mobilizes TorsinA from the membrane of the endoplasmic reticulum (ER) in

1016 response to ER stress and B cell stimulation. Journal of Biological Chemistry, 291(18),

1017 9469-9481. https://doi.org/10.1074/jbc.M115.709337

1018 Zhou, Y., Zhou, B., Pache, L., Chang, M., Khodabakhshi, A. H., Tanaseichuk, O., Benner, C., \&

1019 Chanda, S. K. (2019). Metascape provides a biologist-oriented resource for the analysis of

1020 systems-level datasets. Nature Communications. https://doi.org/10.1038/s41467-019-09234-

10216

1022

1023 


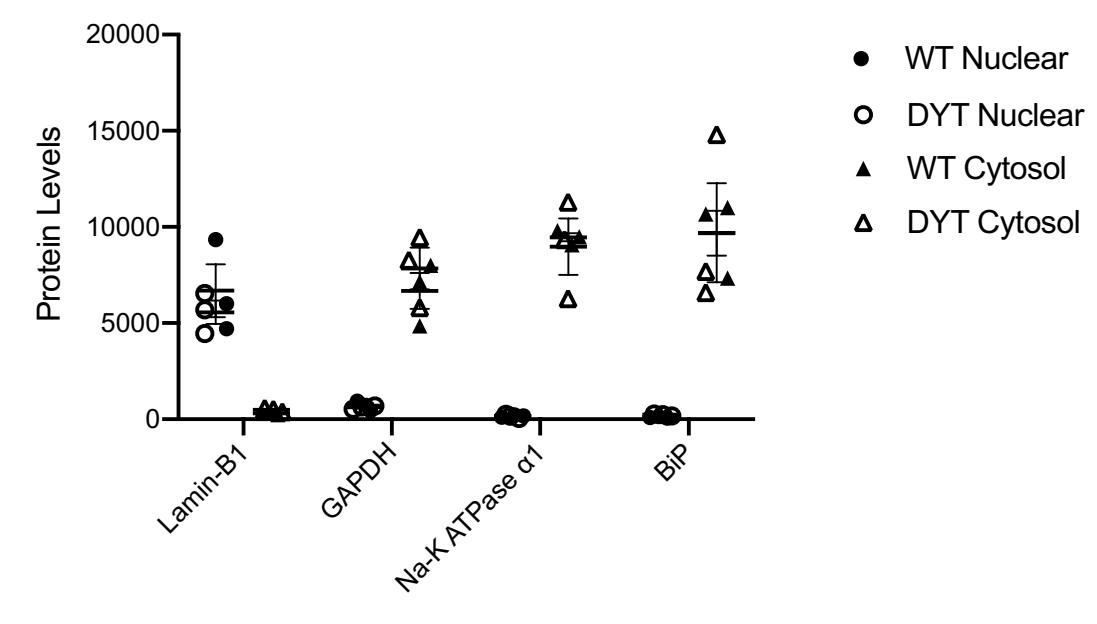

1026 Fig. S1. Quantification of Western blot of WT and DYT-TOR1A samples under basal

1027 conditions. Western blot protein level (a.u.) was quantified by densitometric analysis of the

1028 fluorescent signal. $\mathrm{N}=3$ independent measures per condition. 


\section{A}

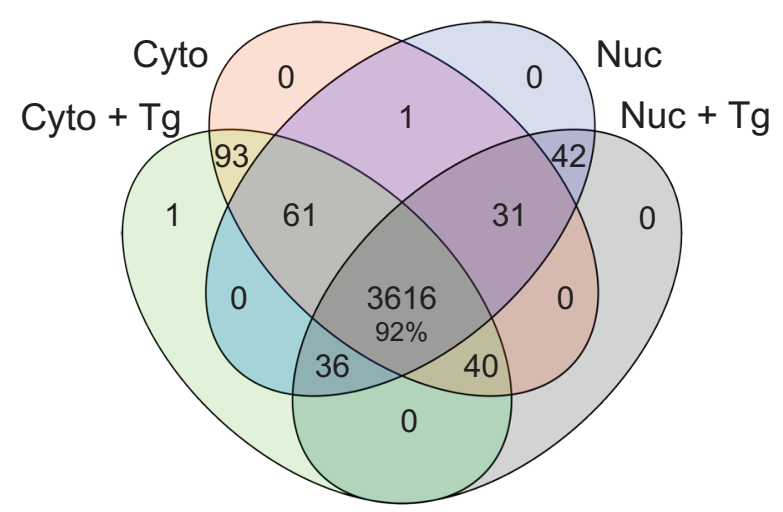

B

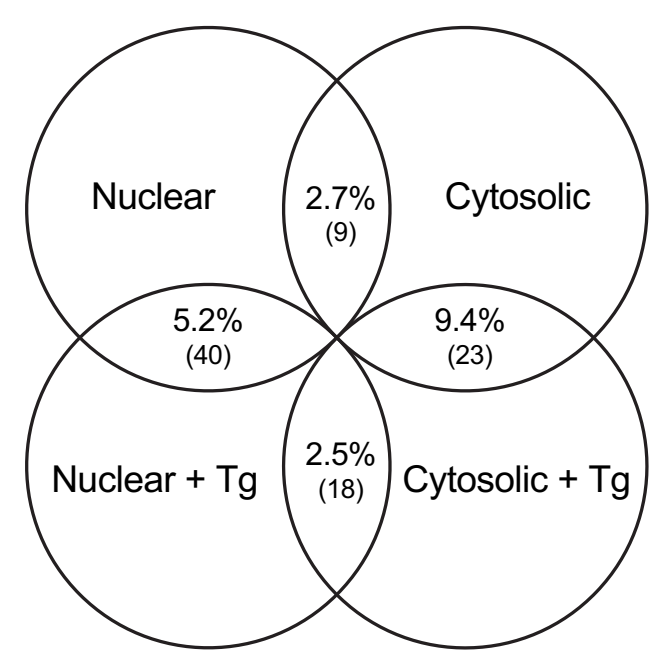

1031 Fig. S2. Overlap of LC/MS/MS identified proteins across experimental conditions. (A) Overlap 1032 of LC/MS/MS identified proteins between nuclear and cytosolic fractions treated with either Tg

1033 or Veh. (B) Pairwise overlap of DYT-disrupted proteins between nuclear and cytosolic fractions 1034 treated with either Tg or Veh. 
A

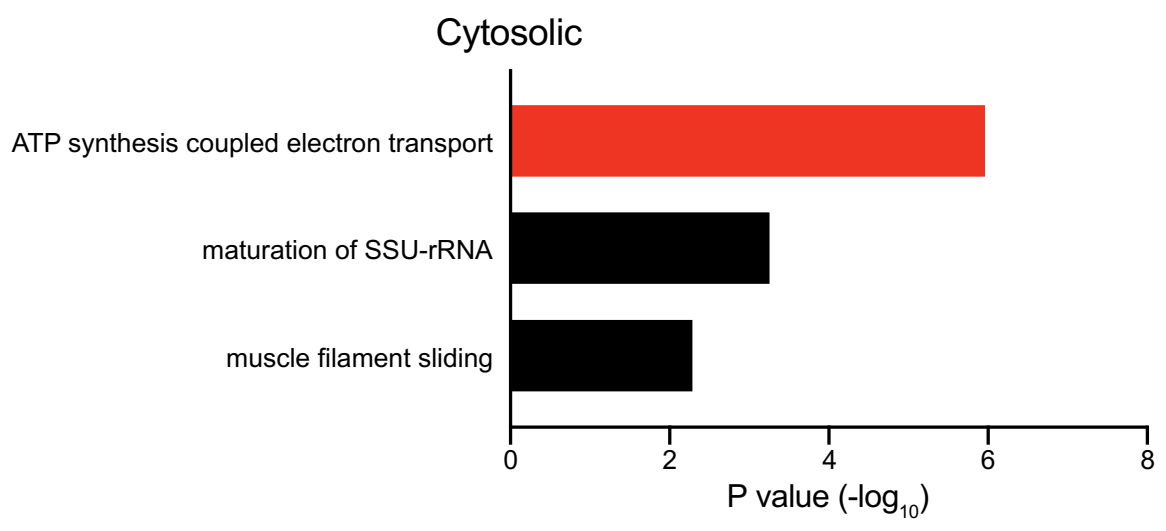

B

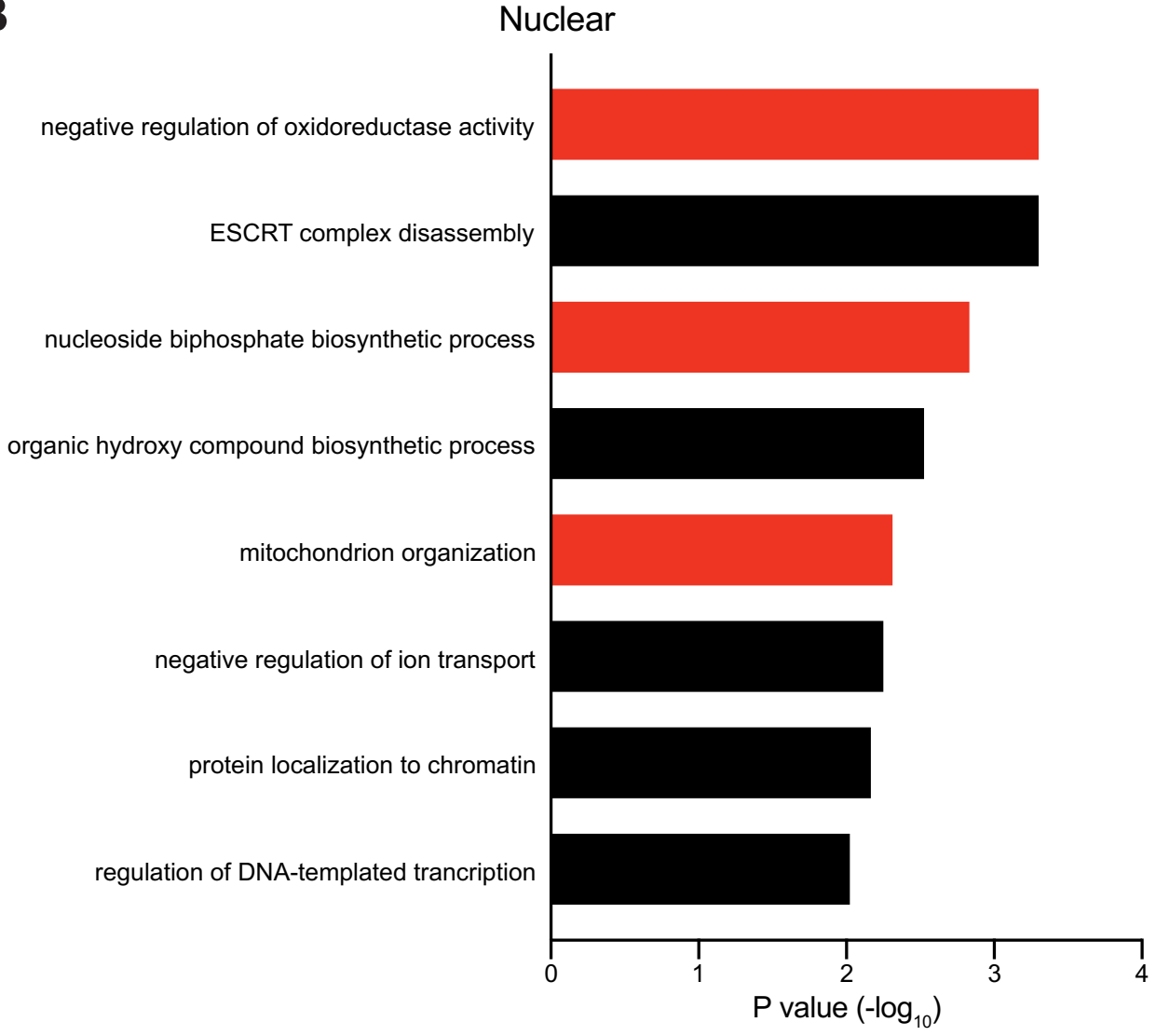

1037 Fig. S3. Gene Ontology analysis of DYT-TOR1A genotype-dependent proteome disruptions. (A)

1038 Gene Ontology analysis of the 152 cytosolic fraction proteins showing DYT-TOR1A/WT or

1039 WT/DYT-TOR1A FC $>1.5$ and $\mathrm{p}<0.05$. (B) Gene Ontology analysis of the 187 nuclear

1040 fraction showing DYT-TOR1A/WT or WT/DYT-TOR1A FC $>1.5$ and $\mathrm{p}<0.05$. Red bars

1041 highlight Gene Ontology terms associated with mitochondrial organization or ATP metabolism. 
A

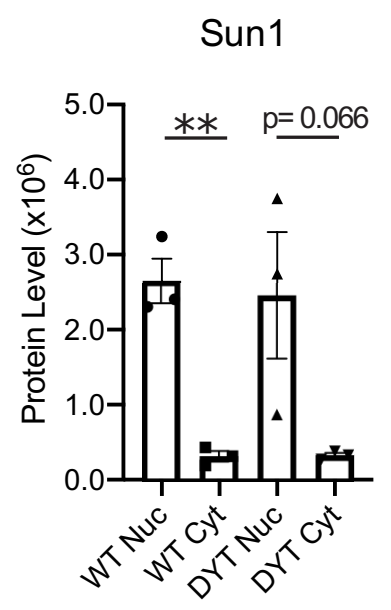

C

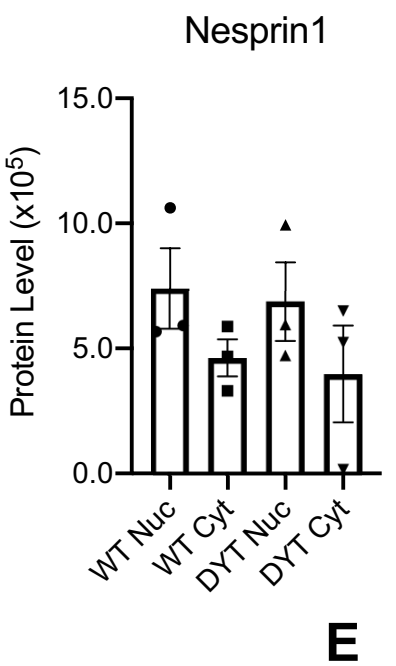

B

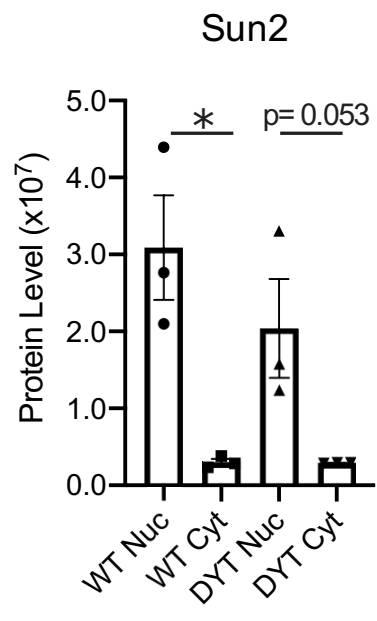

D

Nesprin2

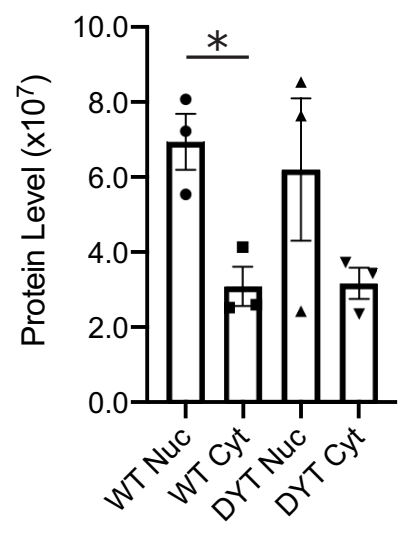

Nesprin3

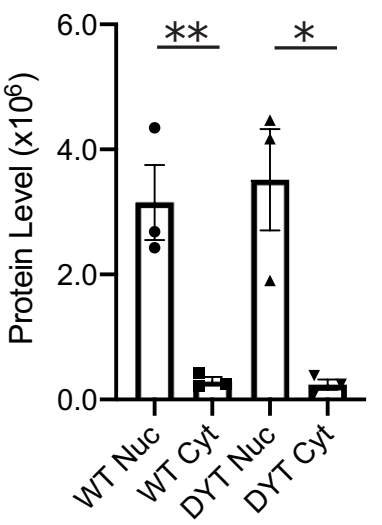


1044 Fig. S4. LINC proteins fractionation across genotype following Veh treatment. Protein levels in

1045 the nuclear and cytosolic fractions of both WT and DYT-TOR1A cell lines for (A) Sun1, (B)

1046 Sun2, (C) Nesprin1, (D) Nesprin2, and (E) Nesprin3. Significance was determined by unpaired t-

1047 test $\left(\mathrm{n}=3\right.$ biological replicates; $\left.{ }^{*} \mathrm{p}<0.05 ; * * \mathrm{p}<0.01\right)$. 
A

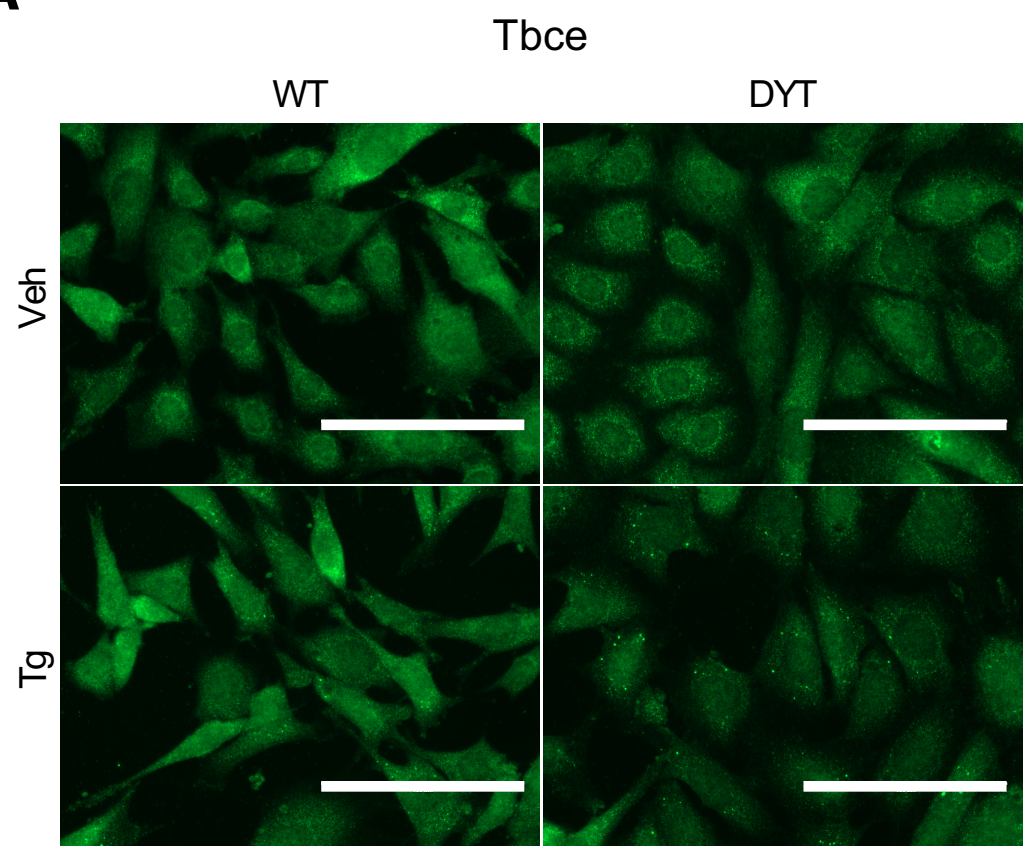

B

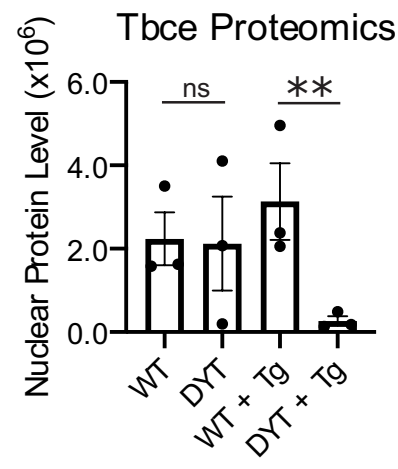

D

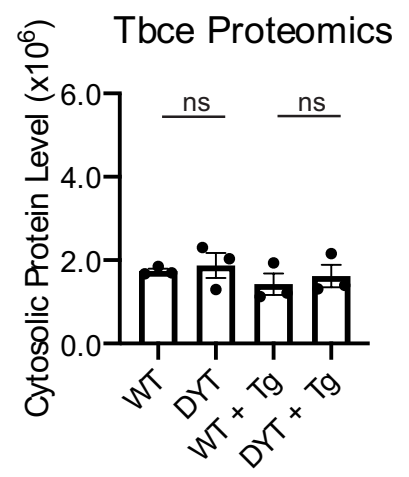

C

Tbce Immunofluorescence

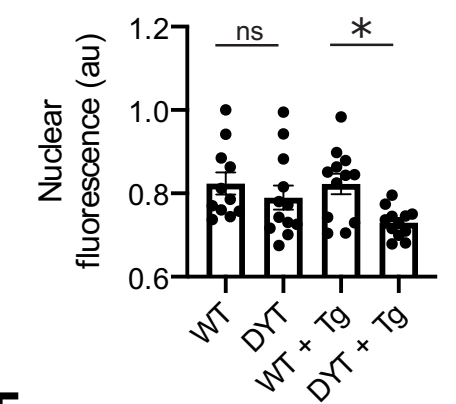

$\mathbf{E}$

Tbce Immunofluorescence

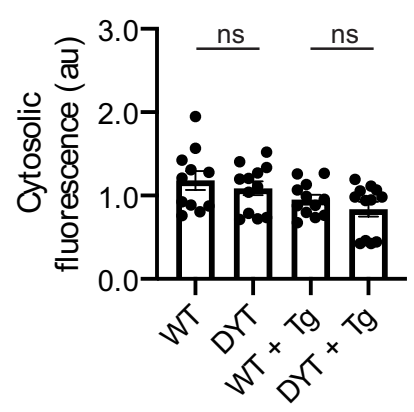

1050 Fig. S5. Quantification of Tbce protein by immunocytochemical staining. (A) Representative 1051 images of Tbce immunofluorescence in WT and DYT-TOR1A MEF lines treated with either 
1052 Veh or Tg. (Scale bar $=100 \mu \mathrm{m})(\mathrm{B})$ Quantitative proteomics data on Tbce protein levels within

1053 the nuclear fractions. (C) Quantification of Tbce immunofluorescence within the nucleus. (D)

1054 Quantitative proteomics data on Tbce protein levels within the cytosolic fractions. (E)

1055 Quantification of Tbce immunofluorescence within the cytosol. For the proteomics data,

1056 significance was determined by unpaired $\mathrm{t}$-test $(\mathrm{n}=3$ biological replicates; $* * \mathrm{p}<0.01)$. For the

1057 immunofluorescence data, significance was determined by unpaired t-test $(\mathrm{n}=12$ biological

1058 replicates with 4 distinct wells being quantified for each of the three unique cell lines per

1059 genotype; $* \mathrm{p}<0.05)$.

1060 
A

Pds5b

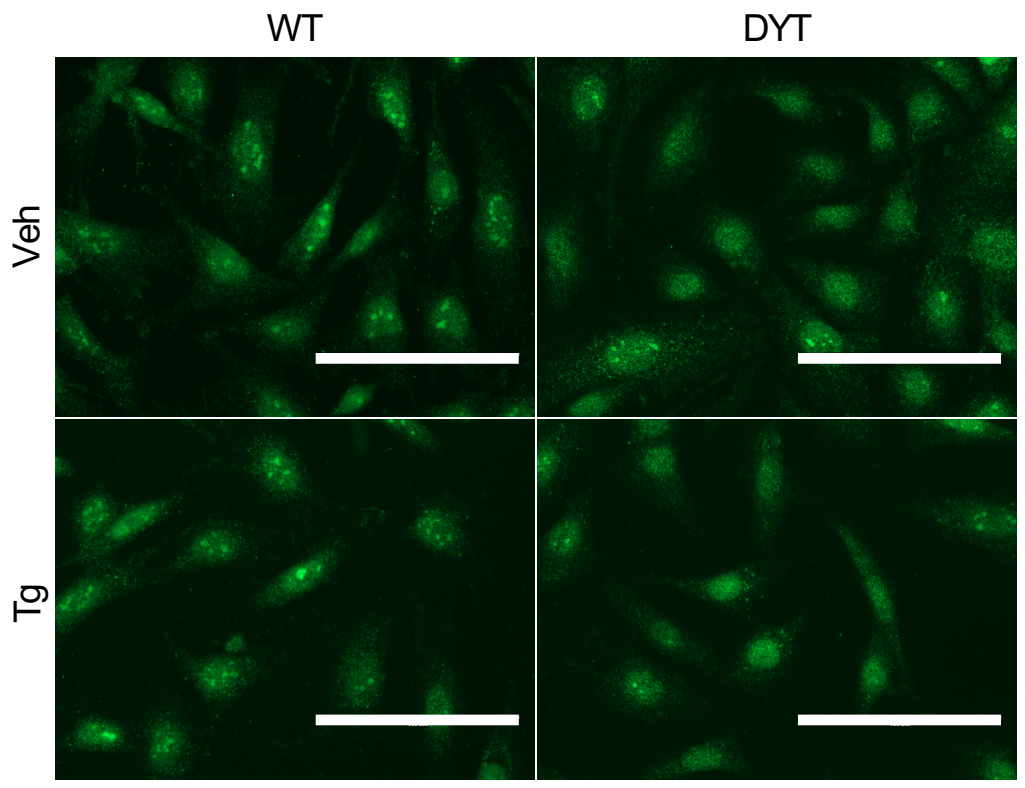

B

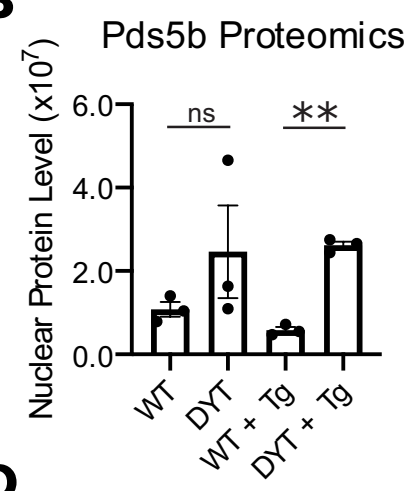

Pds5b Puncta Frequency

C Pds5b Immunofluorescence

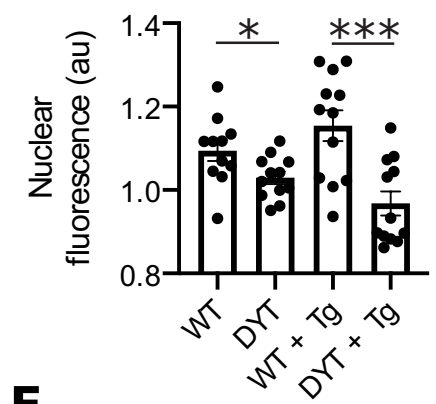

$\mathbf{E}$
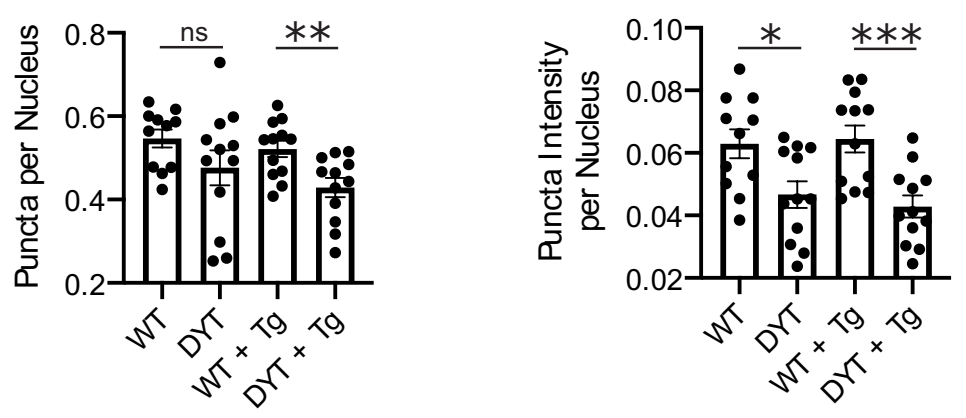

1062 Fig. S6. Quantification of Pds5b protein by immunocytochemical staining. (A) Representative 1063 images of Pds5b immunofluorescence in WT and DYT-TOR1A MEF lines treated with either 
1064 Veh or Tg. (Scale bar $=100 \mu \mathrm{m})(\mathrm{B})$ Quantitative proteomics data on Pds5b protein levels

1065 within the nuclear fractions. (C) Quantification of Pds5b immunofluorescence within the

1066 nucleus. (D) Quantification of Pds5b puncta frequency within the nucleus. (E) Quantification of

1067 cumulative Pds5b puncta intensity within the cell nucleus. For the proteomics data, significance

1068 was determined by unpaired t-test $(\mathrm{n}=3$ biological replicates; $* * \mathrm{p}<0.01)$. For the

1069 immunofluorescence data, significance was determined by unpaired t-test ( $\mathrm{n}=12$ biological

1070 replicates with 4 distinct wells being quantified for each of the three unique cell lines per

1071 genotype; $* \mathrm{p}<0.05, * * * \mathrm{p}<0.001)$. 

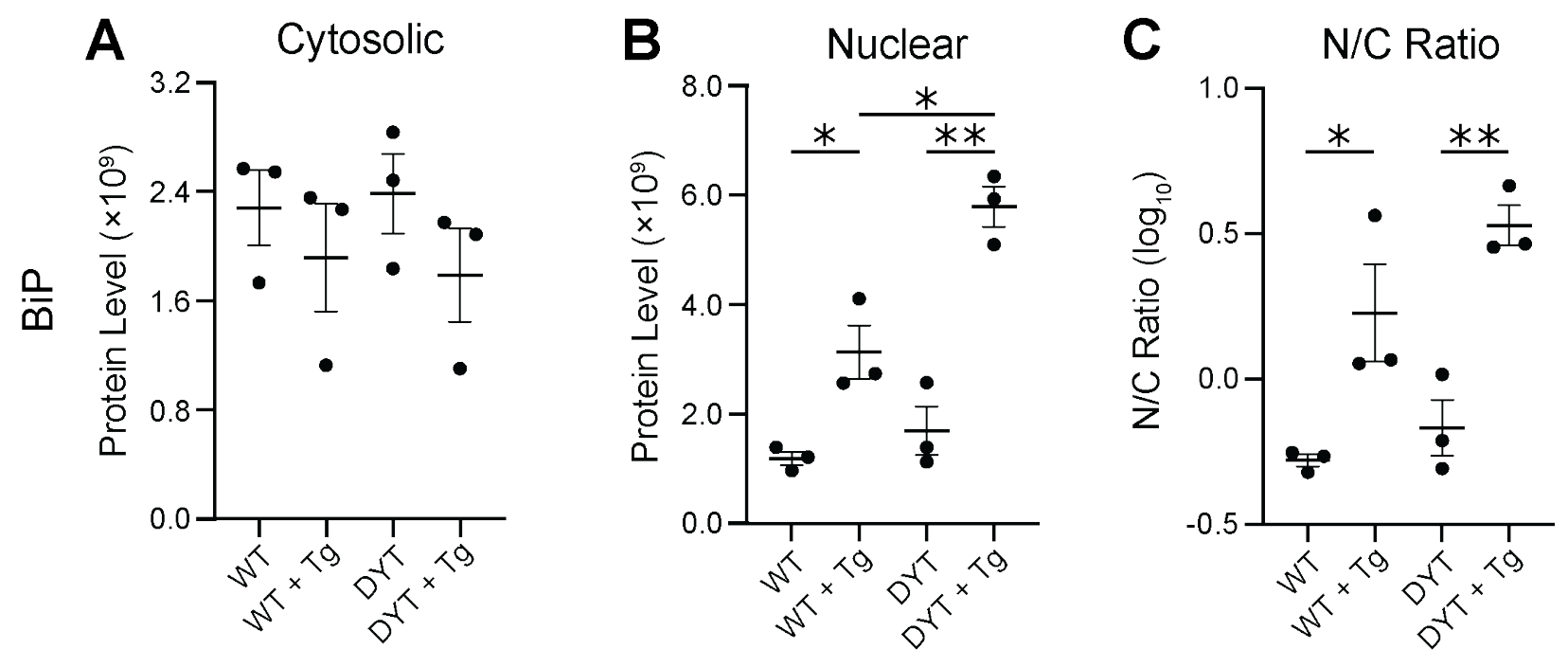

1074

1075 Fig. S7. Genotype and Tg stress effects on subcellular fractionation of BiP. (A-B) LC/MS/MS

1076 quantified relative protein abundance of $\mathrm{BiP}$ in the cytosolic (A) and nuclear (B) fraction. (C)

1077 Ratio of nuclear/cytosolic protein levels. Significance was determined via unpaired t-test $(\mathrm{n}=3$

1078 biological replicates; $\left.{ }^{*} \mathrm{p}<0.05 ; * * \mathrm{p}<0.01\right)$

1079

1080 